\title{
Approximating the Stability Region of a Neural Network with a General Distribution of Delays
}

\author{
R. Jessop ${ }^{\mathrm{a}, 1, *}$, S.A. Campbell ${ }^{\mathrm{b}}$ \\ ${ }^{a}$ Department of Applied Mathematics, University of Waterloo, Waterloo, N2L 3G1, Canada \\ ${ }^{b}$ Department of Applied Mathematics, University of Waterloo, Waterloo, N2L 3G1, Canada
}

\begin{abstract}
We investigate the linear stability of a neural network with distributed delay, where the neurons are identical. We examine the stability of a symmetrical equilibrium point via the analysis of the characteristic equation both when the connection matrix is symmetric and when it is not. We determine a mean delay and distribution independent stability region. We then illustrate a way of improving on this conservative result by approximating the true region of stability when the actual distribution is not known, but some moments or cumulants of the distribution are. Finally, we compare the approximate stability regions with the stability regions in the case of the uniform and gamma distributions. We show that the approximations improve as more moments or cumulants are used, and that the approximations using cumulants give better results than the ones using moments.
\end{abstract}

Keywords: delay differential equations, distributed delay, discrete delay, linear stability, delay independent stability

\section{Introduction}

We consider the following neural network,

$$
C_{k} v_{k}^{\prime}(t)=-\frac{v_{k}(t)}{R_{k}}+\sum_{j=1}^{n} a_{k j} f_{j}\left(v_{j}(t)\right)+I_{k}(t), \quad k=1, \ldots, n,
$$

popularized by Hopfield [21], which is a special case of a more general network first studied by Cohen and Grossberg [8]. In this model, $v_{k}, C_{k}$, and $R_{k}$ denote the voltage, the capacitance, and the resistance of neuron $k$, respectively. Parameter $a_{k j}$ represents the connection strength (or synaptic weight). The exterior current applied to each neuron $k$ is given by $I_{k}$. Function $f_{j}$ models the nonlinear element, which has the property of limiting the output of the signal $v_{j}$. This function $[8,13,21,32]$ is usually taken to be monotonically increasing and differentiable on $(-\infty, \infty)$, with $f_{j}(0)=0, f_{j}^{\prime}(x) \leq f_{j}^{\prime}(0)=\gamma_{j}$ for any $x \in \mathbb{R}$ (where $\gamma_{j}$ is called the gain of neuron $j$ ), and satisfying

$$
\lim _{x \rightarrow \pm \infty} f_{j}(x)= \pm 1, \quad j=1, \ldots, n .
$$

In many biological and physical models, time delays play an important role. They arise for example due to age structure, gestation, maturation, or for biological neural networks, due to the delay in the propagation of the electrochemical signal among neurons. Artificial neural systems also exhibit time delays due to the propagation of the electric signal between nodes and the signal processing inside the nodes. The model (1) assumes that the communication between two nodes is instantaneous. More realistic models of neural networks should include time delays to represent the dependence on past states of the system. Neural networks with time delays were first studied by Grossberg [15, 17, 18, 16]. Marcus and Westervelt [32] introduced time delays into the model (1) to explain

\footnotetext{
${ }^{*}$ Corresponding author

Email address: ramariei@uwaterloo.ca (R. Jessop)

${ }^{1}$ Tel.:1-519-888-4567 ext. 33471, fax:1-519-746-4319
} 
instabilities of equilibria that occurred when implementing this model using real electrical circuits. The resulting model is a system of delay differential equations:

$$
C_{k} v_{k}^{\prime}(t)=-\frac{v_{k}(t)}{R_{k}}+\sum_{j=1}^{n} a_{k j} f_{j}\left(v_{j}\left(t-\tau_{k j}\right)\right)+I_{k}(t), \quad k=1, \ldots, n
$$

where the delay $\tau_{k j}$, represents the time it takes for the signal to travel from neuron $j$ to neuron $k$.

There is a vast literature of neural networks such those in $[15,17,18,16]$ and the model (2) where the delays are fixed (also called discrete). We will not attempt to review this literature in detail, but refer the reader to some recent papers $[22,39,47]$ which contain extensive references to this literature.

As noted above, the time delays in (2) are assumed to be fixed. But many biological and physical events, such as regeneration, recovery period from a disease, or signal conduction, may not take exactly the same time in each instance. Hence the model (2) can be further improved to by including a distribution of delays:

$$
C_{k} v_{k}^{\prime}(t)=-\frac{v_{k}(t)}{R_{k}}+\sum_{j=1}^{n} a_{k j} \int_{0}^{\infty} f_{j}\left(v_{j}(t-u)\right) g_{k j}(u) d u+I_{k}(t), \quad k=1, \ldots, n .
$$

Here the delay $u$, which denotes the time it takes for the signal to travel from neuron $j$ to neuron $k$, occurs with some probability given by the kernel $g_{k j}(u)$, which is a probability density function. Since the time delay must be nonnegative in order to make sense physically and biologically, we take $\int_{0}^{\infty} g_{k j}(u) d u=1$.

Models with distributed delay have been developed mostly in applications to population biology and epidemiology. Examples include the work of Thiel et al. [43] on how distributed delays may abolish complex dynamics present with fixed delays, work on blood cell population models [1, 2, 5], chemostat models [27, 38, 45, 46], epidemic models [3], ecological models $[4,11,19,36]$, and enzyme kinetics [20]. More examples can be found in the references within these papers. The majority of these studies use specific kernels, usually the gamma distribution or the uniform distribution, although some $[3,4,5,11,36]$ obtain results for any distribution. More information on the theory of equations with distributed delay and its application to biological models can be found in several monographs [9, 12, 26, 30, 31].

There are a few papers which have studied neural networks with distributed delays, which we now review. Some papers $[29,37]$ have analyzed low dimensional (e.g. $n=2$ ) networks with specific kernels (gamma distribution with $p=1$ or 2). They study the linear stability of equilibrium points, existence of mean delay induced Hopf bifurcation and stability of the resulting periodic solutions.

The work of Gopalsamy and He [13] investigates the global delay independent region of stability for system (3), where the kernel $g_{k j}$ is a general gamma distribution. They prove the global asymptotic stability of the unique equilibrium point using a Liapunov functional.

Chen [7] considers the neural network

$$
x_{i}^{\prime}(t)=-h_{i}\left(x_{i}(t)\right)+\sum_{j=1}^{n} w_{i j} \int_{-\infty}^{t} g_{i j}(t-u) f_{j}\left(x_{j}(u)\right) d u+I_{i}, \quad i=1 \ldots n
$$

where $h_{i}, i=1 \ldots n$, is differentiable, $f_{j}, j=1 \ldots n$, is Lipschitz, and the kernel $g_{i j}$ is arbitrary. The network (3) is a special case of this model. Using matrix theory and constructing a Liapunov function, he proves the existence, uniqueness and global asymptotic stability of the equilibrium point of (4).

Recent work has extended the Lyapunov function/functional approach to study stability in networks with time varying delays [28, 40], networks with impulses [33] and with stochastic perturbations [42, 44]. The Lyapunov functional approach has also been used to formulate linear matrix inequality (LMI) conditions for stability [35].

In his Master's Thesis, Grégoire-Lacoste [14] investigates the global stability of an equilibrium of system (3). He also performs local stability analysis to determine the boundary of stability of the equilibrium point for the uniform, exponential, triangular, and gamma distributions. The results are compared to the results of the corresponding system with a fixed delay.

In this paper, we analyze the linear stability of the network (3) when the neurons are identical, where the kernel $g$ represents a general distribution. We determine the delay independent region of stability and try to improve on 
this conservative result by approximating the boundary of stability using the first few moments or cumulants of a distribution. We are able to also show that as the mean delay $\tau$ of the distribution becomes larger and larger, the stability region of the network with a distributed delay is also less conservative than the corresponding system with one fixed delay $\tau$. Hence we are able to partially prove the conjecture that a system with distributions of delays is more stable than the corresponding one with a fixed delay.

We note that the distributed delay model (3) includes the fixed delay case, i.e. model (2) corresponds to setting $g_{k j}(u)=\delta\left(u-\tau_{k j}\right)$ in (3), where $\delta$ is the Dirac distribution.

Dividing (3) by $C_{k}$ and assuming that the injected current is constant, we obtain

$$
v_{k}^{\prime}(t)=-\alpha_{k} v_{k}(t)+\sum_{j=1}^{n} w_{k j} \int_{0}^{\infty} f_{j}\left(v_{j}(t-u)\right) g_{k j}(u) d u+F_{k}, \quad k=1, \ldots, n,
$$

where $\alpha_{k}=1 /\left(R_{k} C_{k}\right), w_{k j}=a_{k j} / C_{k}$, and $F_{k}=I_{k} / C_{k}$.

For the rest of the paper, we shall assume that all neurons are identical, hence $\alpha_{k} \equiv \alpha, f_{k}(v) \equiv f(v), \gamma_{k} \equiv \gamma$, and $g_{k j}(u) \equiv g(u)$. Then the above model is reduced to

$$
v_{k}^{\prime}(t)=-\alpha v_{k}(t)+\sum_{j=1}^{n} w_{k j} \int_{0}^{\infty} f\left(v_{j}(t-u)\right) g(u) d u+F_{k}, \quad k=1, \ldots, n
$$

This equation possesses a symmetric equilibrium point, $\mathbf{v}^{*}=\left(v^{*}, v^{*}, \ldots, v^{*}\right)^{T}$, if the following equations are satisfied

$$
\alpha v^{*}-F_{k}=\left(\sum_{j=1}^{n} w_{k j}\right) f\left(v^{*}\right), \quad k=1, \ldots, n .
$$

Given the properties of $f$, we can always guarantee the existence of such an equilibrium by either adjusting the external inputs for a particular connection matrix, or by adjusting the connection strengths when particular external inputs are applied. In particular, if $F_{k}=0, k=1,2, \ldots, n$ the system admits the trivial equilibrium point, $\mathbf{v}^{*}=\mathbf{0}$.

Let $x_{k}(t)=v_{k}(t)-v^{*}$, and $\beta=f^{\prime}\left(v^{*}\right)$. Using (7) and expanding $f\left(v_{j}\right)$ into its Taylor series around $v_{j}=v^{*}$, we obtain the linearization of (6) about $\mathbf{v}^{*}$,

$$
x_{k}^{\prime}(t)=-\alpha x_{k}(t)+\beta \sum_{j=1}^{n} w_{k j} \int_{0}^{\infty} x_{j}(t-u) g(u) d u, \quad k=1, \ldots, n .
$$

We next transform the above equation so that the mean delay

$$
\tau=\int_{0}^{\infty} u g(u) d u
$$

appears explicitly. First we note that as $\tau \rightarrow 0$, then the distribution function $g(u)$ approaches the Dirac distribution $\delta(u)$, since $g(u)$ is nonzero on $(0, \infty)$ and thus, as the mean delay approaches 0 , the entire weight of the distribution gets compressed closer and closer to $u=0$. Hence as $\tau \rightarrow 0$, we recover the non-delayed model

$$
x_{k}^{\prime}(t)=-\alpha x_{k}(t)+\beta \sum_{j=1}^{n} w_{k j} x_{j}(t), \quad k=1, \ldots, n,
$$

which is the linearization about the equilibrium point of system (1) with $n$ identical neurons. Having dealt with the case $\tau=0$, we now restrict to the case $\tau>0$. Making the change of variables $s=t / \tau, v=u / \tau$ and defining $\hat{g}(v)=\tau g(\tau v)$, model (8) becomes

$$
\dot{x}_{k}(s)=-\alpha \tau x_{k}(s)+\beta \tau \sum_{j=1}^{n} w_{k j} \int_{0}^{\infty} x_{j}(s-v) \hat{g}(v) d v, \quad k=1, \ldots, n
$$


where the dot represents the derivative with respect to $s$. We note that the mean of the new distribution $\hat{g}$ is one.

In order to analyze the linear stability of the trivial solution of (10), we compute the characteristic equation associated with the above system. To do so, it is easier to look at the analogous vector form of (10),

$$
\dot{\boldsymbol{x}}(s)=-\alpha \tau \boldsymbol{x}(s)+\beta \tau \boldsymbol{W} \int_{0}^{\infty} \boldsymbol{x}(s-v) \hat{g}(v) d v,
$$

where $\boldsymbol{x}=\left(x_{1}, \ldots, x_{n}\right)^{T}$ and $\boldsymbol{W}$ is an $n \times n$ matrix with the $(k j)^{\text {th }}$ entry given by $w_{k j}$. Let $z_{k}, k=1, \ldots, n$ be the eigenvalues of $\boldsymbol{W}$, then there exists a matrix $\boldsymbol{P}$ such that $\boldsymbol{W}=\boldsymbol{P} \boldsymbol{E} \boldsymbol{P}^{-1}$, where $\boldsymbol{E}$ is an upper triangular matrix with $z_{k}, k=1, \ldots, n$ as diagonal elements. With the change of variables $\boldsymbol{x}=\boldsymbol{P} \boldsymbol{y}$, equation (11) becomes

$$
\dot{\boldsymbol{y}}(s)=-\alpha \tau \boldsymbol{y}(s)+\beta \tau \boldsymbol{E} \int_{0}^{\infty} \hat{g}(v) \boldsymbol{y}(s-v) d v .
$$

Substituting $\boldsymbol{y}=e^{\lambda s} \boldsymbol{c}$ and noticing that the coefficient matrix is upper triangular, the characteristic equation becomes,

$$
\Delta(\lambda)=\prod_{k=1}^{n} \Delta_{k}(\lambda)=\prod_{k=1}^{n}\left(\lambda+\alpha \tau-\beta \tau z_{k} \int_{0}^{\infty} e^{-\lambda v} \hat{g}(v) d v\right)=0 .
$$

Since the characteristic equation is a product of the $\Delta_{k}(\lambda)$ 's, $\lambda$ is a root of $\Delta(\lambda)$ if and only if it is a root of $\Delta_{k}(\lambda)$ for some $k$. Therefore, the linear stability of (10) may be determined by studying the roots of $\Delta_{k}(\lambda), k=1, \ldots, n$. In section 2 we will do this by assuming that the connection matrix $\boldsymbol{W}$ is symmetric, i.e. all its eigenvalues $z_{k}$ are real. In section 3, we consider the case when $\boldsymbol{W}$ is not symmetric, i.e. its eigenvalues may be complex.

\section{Connection Matrix with Real Eigenvalues}

In this section we assume that the eigenvalues of the connection matrix $\boldsymbol{W}$ are real. We start by describing the delay independent region of stability, which we present in the following subsection. This is a conservative result which is useful in practice when one cannot estimate the delay in a system. And then in the following subsection, we improve on this conservative result by approximating the region of stability using just the some statistical properties of a distribution.

\subsection{Distribution independent results}

In this section we will give one result which is independent of all aspects of the distribution and one which is independent of all aspects save the mean delay. The main results of this section generalize to $n$ dimensions the theorems presented in [6] for the scalar case (i.e., (8) with $n=1$ ). The proofs are very similar to the proofs in [6], hence we omit them.

Theorem 1. Assume that $\int_{0}^{\infty} \hat{g}(v) e^{-\lambda v} d v$ is analytic in $\operatorname{Re}(\lambda) \geq 0$. Then the equilibrium point $\mathbf{v}^{*}$ of $(6)$ is locally asymptotically stable if, for each $k=1, \ldots, n$, either

$$
\begin{aligned}
& \text { (1) }\left|z_{k}\right|<\frac{\alpha}{\beta}, \\
& \text { or } \\
& \text { (2) }-\frac{1}{\beta \tau}<z_{k} \leq-\frac{\alpha}{\beta} .
\end{aligned}
$$

We note that if all $z_{k}, k=1, \ldots, n$ satisfy the condition in (1), then the equilibrium $\mathbf{v}^{*}$ of (6) is locally asymptotically stable for any value of the mean delay $\tau$ and for all distributions $\hat{g}(v)$. We will call this the delay independent stability region. The following result determines a region in the parameter space where the equilibrium point is unstable for any distribution.

Theorem 2. The equilibrium point $\mathbf{v}^{*}$ of (6) is unstable if at least one $z_{k}, k=1, \ldots, n$, satisfies $z_{k}>\alpha / \beta$. 


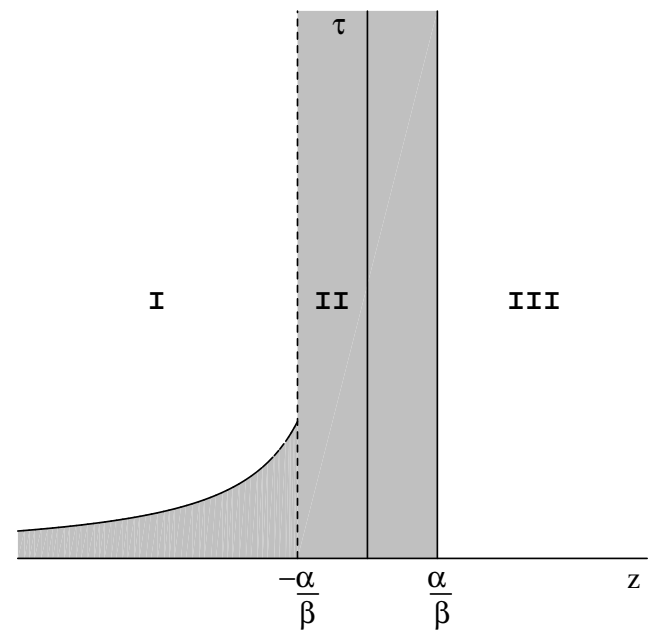

Figure 1: Illustration of the distribution independent stability results described by Theorems 1 and 2. (I) No distribution independent stability results are known for this region. (II) Region of stability described by Theorem 1: all $z_{k}$ 's must lie to the right of the curve $-1 /(\beta \tau)$ and be less than $-\alpha / \beta$, or have norm less than $\alpha / \beta$. (III) Region of instability described by Theorem 2: at least one of the $z_{k}$ 's must be greater than $\alpha / \beta$.

The results of Theorems 1 and 2 are illustrated in Figure 1. The stability region in the shaded area is a conservative result and is independent of the distribution, save the mean delay.

In practice the results of Theorem 1 are useful only if one is able to compute $\beta=f^{\prime}\left(v^{*}\right)$. This might not be trivial since one has to solve the nonlinear system (7). But since we know that $\beta \leq f^{\prime}(0)=\gamma$, we can use this to obtain the following corollary to Theorem 1:

Corollary 1. Assume that $\int_{0}^{\infty} \hat{g}(v) e^{-\lambda v} d v$ is analytic in $\operatorname{Re}(\lambda) \geq 0$. Then the equilibrium point $\mathbf{v}^{*}$ of $(6)$ is locally asymptotically stable if, for each $k=1, \ldots, n$, either

$$
\begin{aligned}
& \text { (1) }\left|z_{k}\right|<\frac{\alpha}{\gamma}, \\
& \text { or } \\
& \text { (2) }-\frac{1}{\gamma \tau}<z_{k} \leq-\frac{\alpha}{\gamma} .
\end{aligned}
$$

The results of the above corollary and their comparison to Theorem 1 are presented in Figure 2 . Since $\beta \leq \gamma$, it is clear that the stability result presented in Corollary 1 is more conservative than the result of Theorem 1, but it might be more useful in practice since one only needs to know the neuron gain, $\gamma=f^{\prime}(0)$.

Theorem 1 gives an approximation to the stability region of the equilibrium point. The exact stability region has a boundary consisting of points in the parameter space where the characteristic equation (12) has roots with zero real part. We now investigate this boundary.

Note that for any distribution, the characteristic equation has a zero root if for at least one $k, \beta z_{k}=\alpha$. To further define the boundary of stability, we need to determine where the characteristic equation has a pair of pure imaginary roots, $\lambda= \pm i \omega$. We consider the most generic case: suppose that for one $k, \Delta_{k}(\lambda)$ has a pair of pure imaginary roots, i.e.

$$
i \omega+\alpha \tau-\beta \tau z_{k} \int_{0}^{\infty} e^{-i \omega v} \hat{g}(v) d v=0 .
$$

Separating this into real and imaginary parts we find

$$
\begin{aligned}
\alpha \tau & =\beta \tau z_{k} \int_{0}^{\infty} \cos (\omega v) \hat{g}(v) d v \stackrel{\text { def }}{=} \beta \tau z_{k} C(\omega), \\
-\omega & =\beta \tau z_{k} \int_{0}^{\infty} \sin (\omega v) \hat{g}(v) d v \stackrel{\text { def }}{=} \beta \tau z_{k} S(\omega) .
\end{aligned}
$$




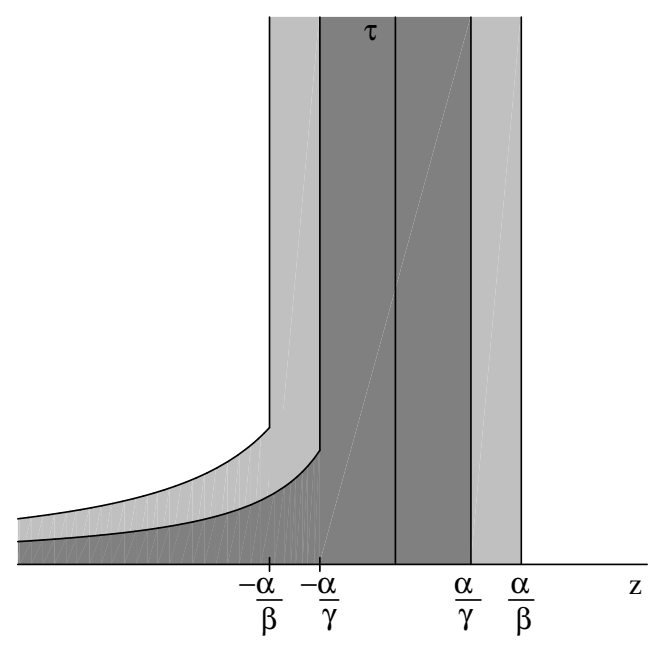

Figure 2: Comparison between the stability results described by Corollary 1 and the ones presented in Theorem 1. The stability region guaranteed by Corollary 1 (the dark gray region) is more conservative than the one guaranteed by Theorem 1 (the light gray region), but it is easier to obtain since it is only dependent on the neuron gain $\gamma$.

\section{Consider}

$$
z=\frac{\alpha}{\beta C(\omega)}, \quad \tau=-\frac{\omega C(\omega)}{\alpha S(\omega)},
$$

for all $\omega>0$ such that $C(\omega)$ and $S(\omega)$ are nonzero. Equations (15) represent curves in the $z \tau$-plane parameterized by $\omega$. We then choose the curve which is the closest to the $\tau$-axis. If all $z_{k}, k=1, \ldots, n$ lie on or below this curve then the equilibrium point of (10) is stable. Hence the curve described by (15) and closest to the $\tau$-axis forms part of the boundary of stability.

We next determine whether the real part of the eigenvalue increases or decreases as we cross the lines in (15). Taking the derivative of $\tau$ in (15) with respect to $\omega$ we obtain

$$
\frac{d \tau}{d \omega}=-\frac{1}{\alpha S(\omega)}\left(C(\omega)+\omega \frac{C^{\prime}(\omega) S(\omega)-S^{\prime}(\omega) C(\omega)}{S(\omega)}\right) .
$$

Using the definitions in (14), we have

$$
\Delta_{k}(i \omega)=i \omega+\alpha \tau-\beta \tau z_{k}(C(\omega)-i S(\omega))=0 .
$$

Next we compute the rate of change of the real part of $\lambda$ with respect to $z_{k}$. Using (17), we first compute

$$
\left.\frac{\partial \Delta}{\partial z_{k}}\right|_{\lambda=i \omega}=-\beta \tau[C(\omega)-i S(\omega)] \prod_{\substack{r=1 \\ r \neq k}}^{n}\left(i \omega+\alpha \tau-\beta \tau z_{r}(C(\omega)-i S(\omega))\right),
$$

and

$$
\left.\frac{\partial \Delta}{\partial \lambda}\right|_{\lambda=i \omega}=\left[1+\beta \tau z_{k}\left(S^{\prime}(\omega)+i C^{\prime}(\omega)\right)\right] \prod_{\substack{r=1 \\ r \neq k}}^{n}\left(i \omega+\alpha \tau-\beta \tau z_{r}(C(\omega)-i S(\omega))\right) .
$$


Therefore

$$
\begin{aligned}
\left.\frac{d \operatorname{Re}(\lambda)}{d z_{k}}\right|_{\lambda=i \omega} & =-\operatorname{Re}\left(\frac{\partial \Delta_{k}}{\partial \beta z_{k}} /\left.\frac{\partial \Delta_{k}}{\partial \lambda}\right|_{\lambda=i \omega}\right) \\
& =-\operatorname{Re}\left(\frac{-\beta \tau(C(\omega)-i S(\omega))}{1+\beta \tau z_{k}\left(S^{\prime}(\omega)+i C^{\prime}(\omega)\right)}\right) \\
& =\frac{\beta \tau}{H^{2}(\omega)}\left(C(\omega)+\omega \frac{C^{\prime}(\omega) S(\omega)-S^{\prime}(\omega) C(\omega)}{S(\omega)}\right),
\end{aligned}
$$

where $H^{2}(\omega)=\left(1+\beta \tau z_{k} S^{\prime}(\omega)\right)^{2}+\left(\beta \tau z_{k} C^{\prime}(\omega)\right)^{2}$ is a positive function of $\omega$ and we have used that $\beta \tau z_{k}=$ $-\omega / S(\omega)$ from (15). Comparing with (16) we see that

$$
\left.\frac{d \operatorname{Re}(\lambda)}{d z_{k}}\right|_{\lambda=i \omega}=\frac{\alpha \omega}{z_{k} H^{2}(\omega)} \frac{d \tau}{d \omega} .
$$

Thus whether the number of eigenvalues with positive real part is increasing or decreasing as $z_{k}$ is increased through a point on one of the curves defined by (15) depends on the sign of $z_{k}$ and whether $\tau$ is an increasing or decreasing function of $\omega$ at the point.

To further characterize the stability region we need to use information from the distribution. In the next subsection we will show how one may find a better approximation to the region of stability than that given by Theorem 1 (shaded area in Figure 1) by using more information from the distribution. In subsection 2.3 we will compare the various approximations with the exact stability region determined with full knowledge of the distribution. We note that it is only necessary to consider $z_{k}<0$, given the results of Theorems 1 and 2.

\subsection{Approximating the boundary of the stability region}

In practice, we may not know the exact distribution of delays in a system, however, we may be able to determine some statistical properties of the distribution. In the following we show how to approximate the true boundary of stability, using only the first few moments or cumulants of the distribution.

To begin, recall that the moments and cumulants of a distribution can by defined and computed using the momentcumulant generating function

$$
\phi(t)=\int_{0}^{\infty} e^{i t v} \hat{g}(v) d v .
$$

The moments $m_{n}$ and the cumulants $\kappa_{n}$ are then given by [34]

$$
\left.\frac{d^{n}}{d t^{n}} \phi(t)\right|_{t=0}=i^{n} m_{n} \quad \text { and }\left.\quad \frac{d^{n}}{d t^{n}} \ln \phi(t)\right|_{t=0}=i^{n} \kappa_{n} .
$$

We note that, for any distribution, $m_{0}=\phi(0)=1$ and $\kappa_{0}=\ln \phi(0)=0$. Further, the first cumulant is equal to the first moment and represents the mean of a distribution. The second cumulant is equal to the variance of a distribution, and the second moment is the sum of the variance and the square of the mean. In our case $\kappa_{1}=m_{1}=1$, since the mean of our normalized distribution $\hat{g}(v)$ is one. The higher cumulants can be obtained recursively from the moments using Faá di Bruno's formula [24],

$$
\begin{aligned}
& \kappa_{2}=m_{2}-m_{1}^{2}, \\
& \kappa_{3}=m_{3}-3 m_{1} m_{2}+2 m_{1}^{3},
\end{aligned}
$$

and so on. Expanding $\phi(t)$ in its Taylor series around $t=0$, we have

$$
\phi(t)=\left.\sum_{n=0}^{\infty} \frac{d^{n}}{d t^{n}} \phi(t)\right|_{t=0} \frac{t^{n}}{n !}=\sum_{n=0}^{\infty} i^{n} m_{n} \frac{t^{n}}{n !} .
$$

Similarly, expanding $\ln \phi(t)$ we obtain

$$
\ln \phi(t)=\left.\sum_{n=0}^{\infty} \frac{d^{n}}{d t^{n}} \ln \phi(t)\right|_{t=0} \frac{t^{n}}{n !}=\sum_{n=0}^{\infty} i^{n} \kappa_{n} \frac{t^{n}}{n !} .
$$


Substituting $t=-\omega$, from (19), (21) and (22), we obtain

$$
\int_{0}^{\infty} e^{-i \omega v} \hat{g}(v) d v=\sum_{n=0}^{\infty}(-1)^{n} i^{n} m_{n} \frac{\omega^{n}}{n !}=\exp \left\{\sum_{n=0}^{\infty}(-1)^{n} i^{n} \kappa_{n} \frac{\omega^{n}}{n !}\right\} .
$$

From the definitions of $C(\omega)$ and $S(\omega)$, we have

$$
C(\omega)=\operatorname{Re}\left(\int_{0}^{\infty} e^{-i \omega v} \hat{g}(v) d v\right) \quad \text { and } \quad S(\omega)=-\operatorname{Im}\left(\int_{0}^{\infty} e^{-i \omega v} \hat{g}(v) d v\right) .
$$

From (23) we obtain the following approximations for $C(\omega)$ and $S(\omega)$ in terms of the moments,

$$
C(\omega) \approx \sum_{n=0}^{M} \frac{(-1)^{n} \omega^{2 n}}{(2 n) !} m_{2 n} \quad \text { and } \quad S(\omega) \approx \sum_{n=0}^{N} \frac{(-1)^{n} \omega^{2 n+1}}{(2 n+1) !} m_{2 n+1} .
$$

The first two approximations using moments are show in Table 1. We note that the first approximation using moments,

Table 1: Approximations for $C(\omega)$ and $S(\omega)$ using moments.

\begin{tabular}{|c||c|c|}
\hline$(M, N)$ & $C(\omega)$ & $S(\omega)$ \\
\hline$(1,0)$ & $1-\frac{m_{2}}{2} \omega^{2}$ & $\omega$ \\
\hline$(1,1)$ & $1-\frac{m_{2}}{2} \omega^{2}$ & $\omega-\frac{m_{3}}{6} \omega^{3}$ \\
\hline
\end{tabular}

i.e. substituting the values of $C(\omega)$ and $S(\omega)$ from the first row of Table 1 into (15), recovers the results of Theorem 1 , i.e., that the approximate boundary of the stability region is given by

$$
\tau=-\frac{1}{\beta z_{k}}
$$

Using (23) again, we can also obtain the following approximations for $C(\omega)$ and $S(\omega)$ in terms of cumulants,

$$
C(\omega) \approx \exp \left\{\sum_{n=0}^{M} \frac{(-1)^{n} \omega^{2 n}}{(2 n) !} \kappa_{2 n}\right\} \cos \left\{\sum_{n=0}^{N} \frac{(-1)^{n} \omega^{2 n+1}}{(2 n+1) !} \kappa_{2 n+1}\right\}
$$

and

$$
S(\omega) \approx \exp \left\{\sum_{n=1}^{M} \frac{(-1)^{n} \omega^{2 n}}{(2 n) !} \kappa_{2 n}\right\} \sin \left\{\sum_{n=1}^{N} \frac{(-1)^{n} \omega^{2 n+1}}{(2 n+1) !} \kappa_{2 n+1}\right\} .
$$

The first two approximations using cumulants can be seen in Table 2. We note that the first approximation using

Table 2: Approximations for $C(\omega)$ and $S(\omega)$ using cumulants.

\begin{tabular}{|c||c|c|}
\hline$(M, N)$ & $C(\omega)$ & $S(\omega)$ \\
\hline$(0,1)$ & $\cos (\omega)$ & $\sin (\omega)$ \\
\hline$(1,1)$ & $\exp \left(-\kappa_{2} \frac{\omega^{2}}{2}\right) \cos (\omega)$ & $\exp \left(-\kappa_{2} \frac{\omega^{2}}{2}\right) \sin (\omega)$ \\
\hline
\end{tabular}

cumulants recovers the results for the corresponding system with one fixed delay $\tau$ (i.e. the linear stability region of the equilibrium point of system (2) with $n$ identical neurons). The stability boundary of the scalar model with one 
fixed delay is analyzed in [10]. Generalizing their result to the $n$ dimensional model, we obtain the stability boundary for the equilibrium point $v^{*}$ of the system with one fixed delay (2),

$$
\tau=\frac{1}{\sqrt{\beta^{2} z_{k}^{2}-\alpha^{2}}} \arccos \left(\frac{\alpha}{\beta z_{k}}\right), \quad z_{k}<-\frac{\alpha}{\beta} .
$$

Since $\arccos \left(\alpha /\left(\beta z_{k}\right)\right)>1$ for $z_{k}<-\alpha / \beta$, from (26) and (29), we can conclude that the the first approximation using cumulants always lies above the first approximation using moments. We also notice that the curve described by (29) has a vertical asymptote at $z_{k}=-\alpha / \beta$ and thus it never enters the delay independent region of stability, $\left|z_{k}\right| \leq \alpha / \beta$ described by result (1) of Theorem 1. On the other hand, the first approximation using moments given by (26) has $z_{k}=0$ as its vertical asymptote, and does enter the delay independent stability region at $z_{k}=-\alpha / \beta$.

Substituting the values of $C(\omega)$ and $S(\omega)$ from the second row of Table 2 into (15), we obtain the second approximation using cumulants,

$$
\tau=-\frac{\omega e^{\kappa_{2} \omega^{2} / 2}}{\beta z_{k} \sin (\omega)},
$$

where $\omega \in(\pi / 2, \pi)$ in order to obtain the curve closest to the $\tau$-axis. But as seen in (20), the second cumulant represents the variance of a distribution, i.e. $\kappa_{2}=\sigma^{2}$, where $\sigma$ is the standard deviation, and thus $\kappa_{2}$ is always nonnegative. Therefore, comparing the above equation to (26), we can also conclude that second approximation using cumulants always lies above first approximation using moments.

In the next two sections we compute several approximations for the uniform and gamma distributions and compare them to the true region of stability.

\subsection{Verifying the approximations for the uniform distribution}

In this section we apply the approximation procedure we derived in the previous section to the uniform distribution, thus determining approximations for the boundary of the region of stability. We will then compare these approximations with the true boundary derived from the characteristic equation. The normalized uniform distribution is given by

$$
\hat{g}(v)=\left\{\begin{array}{lc}
\frac{1}{\rho}, & \text { if } v \in\left[1-\frac{\rho}{2}, 1+\frac{\rho}{2}\right] \\
0, & \text { elsewhere }
\end{array}\right.
$$

The moments are given by

$$
m_{n}=\frac{1}{(n+1) \rho}\left[\left(1+\frac{\rho}{2}\right)^{n+1}-\left(1-\frac{\rho}{2}\right)^{n+1}\right] .
$$

Using this and the recursive formula in (20), we compute the first few moments and cumulants for $\rho=1$ and $\rho=2$, as shown in Table 3.

Table 3: Moments and cumulants of the uniform distribution.

\begin{tabular}{|c||c|c|c|c||c|c|c|c|}
\hline$\rho$ & $m_{0}$ & $m_{1}$ & $m_{2}$ & $m_{3}$ & $\kappa_{0}$ & $\kappa_{1}$ & $\kappa_{2}$ & $\kappa_{3}$ \\
\hline 1 & 1 & 1 & $13 / 12$ & $5 / 4$ & 0 & 1 & $1 / 12$ & 0 \\
\hline 2 & 1 & 1 & $4 / 3$ & 2 & 0 & 1 & $1 / 3$ & 0 \\
\hline
\end{tabular}

Using (30), we determine the exact form for $C(\omega)$ and $S(\omega)$,

$$
\begin{aligned}
& C(\omega)=\frac{1}{\rho} \int_{1-\rho / 2}^{1+\rho / 2} \cos (\omega v) d v=\frac{2 \cos (\omega) \sin (\rho \omega / 2)}{\rho \omega}, \\
& S(\omega)=\frac{1}{\rho} \int_{1-\rho / 2}^{1+\rho / 2} \sin (\omega v) d v=\frac{2 \sin (\omega) \sin (\rho \omega / 2)}{\rho \omega} .
\end{aligned}
$$




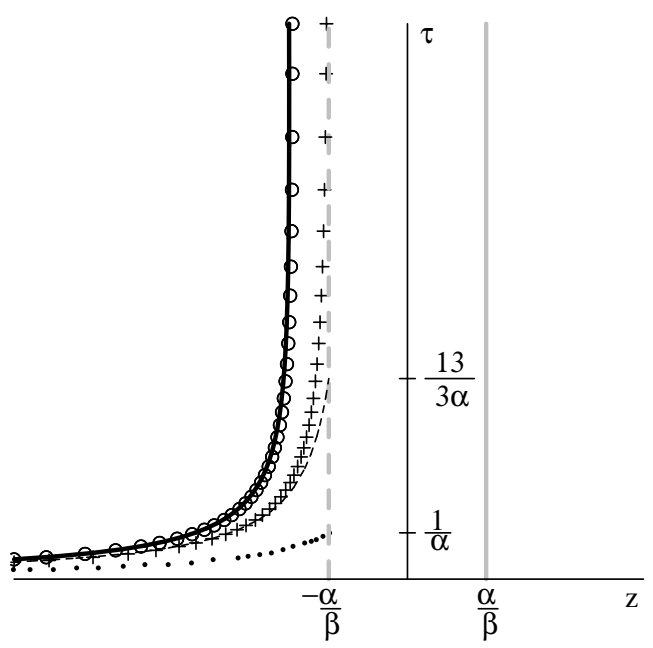

(a) $\rho=1$

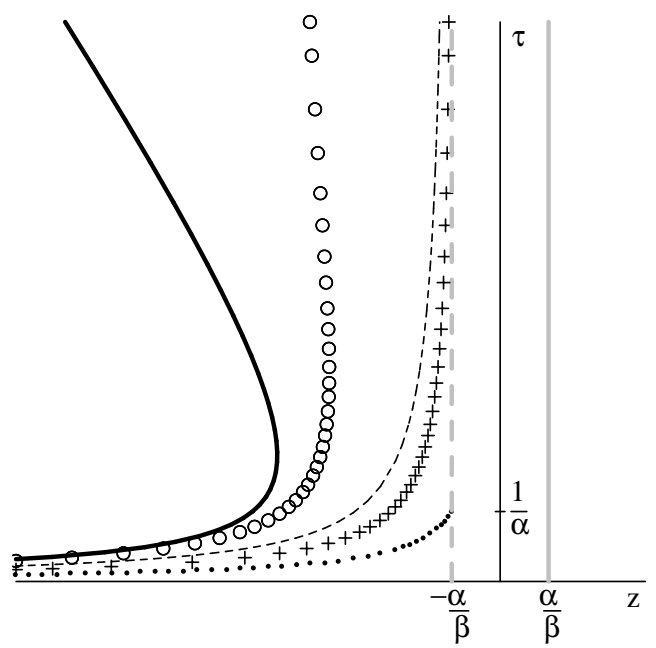

(b) $\rho=2$

Figure 3: Stability region for the uniform distribution with $\rho=1$ and $\rho=2$. The region of distribution independent stability lies between the solid and dashed gray lines. The true region of stability lies between the solid gray line and the solid black curve. The dotted and dash curves show the first and second approximations using the moments, respectively. The curves depicted by crosses and circles represent the first and second approximation using the cumulants, respectively.

Substituting these into equations (15), we obtain the true boundary of stability in the $z \tau$-plane, as shown by the black solid curve in Figures 3(a) and 3(b) (detail on how these curves are obtained is presented in [6]). Thus the true region of stability lies between the solid gray line and the solid black curve, where the region of distribution independent stability lies between the solid and dashed gray lines. Substituting the approximations using moments, i.e. equations (25), into (15) we get approximations to the true boundary of stability depicted as the dotted and dash lines in Figures 3(a) and 3(b). And finally, the first two approximations using cumulants (curves depicted by crosses and circles) in Figures 3(a) and 3(b) are obtained by substituting (27) and (28) into (15).

\subsection{Verifying the approximations for the gamma distribution}

In this section we will apply the approximations to the gamma distribution and compare these approximations with the true boundary of stability derived from the characteristic equation. The normalized gamma distribution is given by

$$
\hat{g}(v)=\frac{p^{p} v^{p-1} e^{-p v}}{(p-1) !}
$$

and the $n^{\text {th }}$ moment is

$$
m_{n}=p^{-n} \frac{(n+p-1) !}{(p-1) !} .
$$

Using this and (20), we obtain the first few moments and cumulants for $p=2$ and $p=3$, which are shown in Table 4. We here note that for $p=1$, the true region of stability is given by the entire plane to the left of the line $z_{k}=\alpha / \beta$ and that the second approximation using moments and the first two approximations using cumulants recover this true region of stability.

Using the identity $\int_{0}^{\infty} v^{n} e^{-p v} d v=n ! / p^{n+1}$, we obtain the exact expression for $C(\omega)$ and $S(\omega)$,

$$
\begin{aligned}
& C(\omega)=\operatorname{Re}\left(\frac{p^{p}}{(p-1) !} \int_{0}^{\infty} v^{p-1} e^{-(p+i \omega) v} d v\right)=\left(1+\frac{\omega^{2}}{p^{2}}\right)^{-p} \operatorname{Re}\left(1-\frac{i \omega}{p}\right)^{p}, \\
& S(\omega)=-\operatorname{Im}\left(\frac{p^{p}}{(p-1) !} \int_{0}^{\infty} v^{p-1} e^{-(p+i \omega) v} d v\right)=-\left(1+\frac{\omega^{2}}{p^{2}}\right)^{-p} \operatorname{Im}\left(1-\frac{i \omega}{p}\right)^{p} .
\end{aligned}
$$


Table 4: Moments and cumulants of the gamma distribution.

\begin{tabular}{|c||c|c|c|c||c|c|c|c|}
\hline$p$ & $m_{0}$ & $m_{1}$ & $m_{2}$ & $m_{3}$ & $\kappa_{0}$ & $\kappa_{1}$ & $\kappa_{2}$ & $\kappa_{3}$ \\
\hline 2 & 1 & 1 & $3 / 2$ & 3 & 0 & 1 & $1 / 2$ & $1 / 2$ \\
\hline 3 & 1 & 1 & $4 / 3$ & $20 / 9$ & 0 & 1 & $1 / 3$ & $2 / 9$ \\
\hline
\end{tabular}

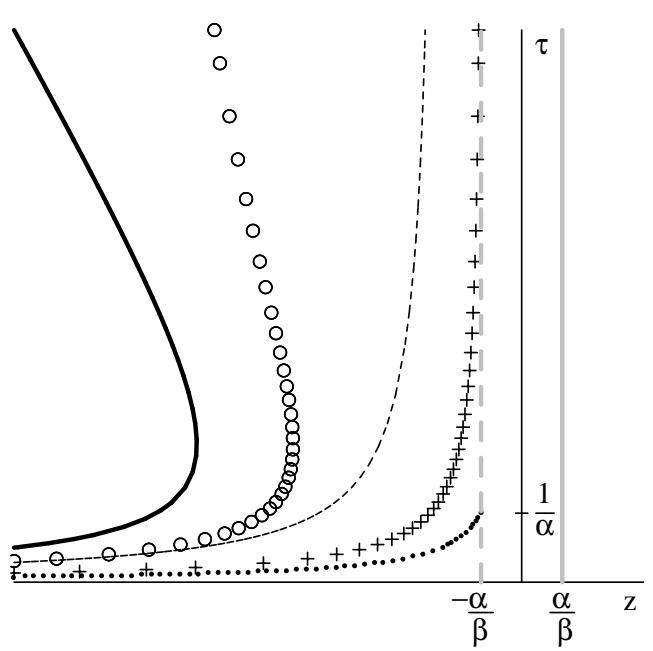

(a) $p=2$

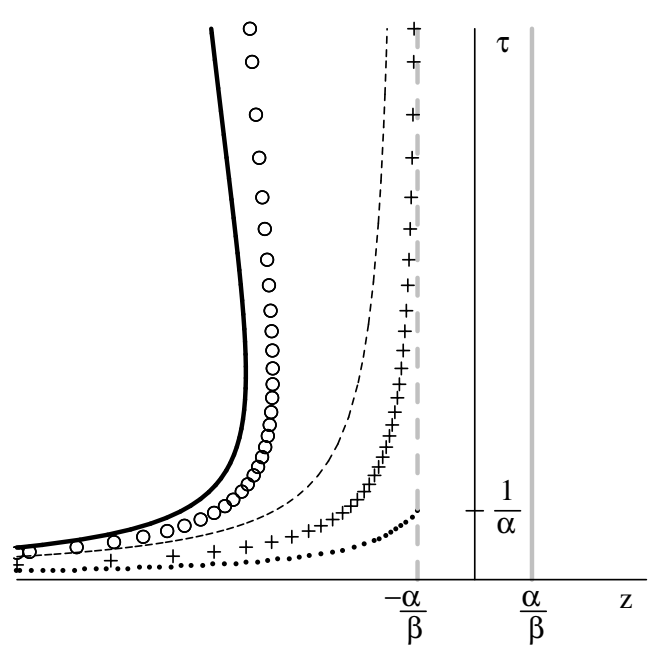

(b) $p=3$

Figure 4: Stability region for the gamma distribution with $p=2$ and $p=3$. The region of distribution independent stability lies between the solid and dashed gray lines. The true region of stability lies between the solid gray line and the solid black curve. The dotted and dash curves show the first and second approximations using the moments, respectively. The curves depicted by crosses and circles represent the first and second approximation using the cumulants, respectively.

Substituting the exact expressions for $C(\omega)$ and $S(\omega)$ into equations (15), we obtain the true boundary of stability in the $z \tau$-plane, as shown by the black solid curve in Figures 4(a) for $p=2$ and 4(b) for $p=3$ (detail on how these curves are obtained is presented in [6]). Thus the true region of stability lies between the solid gray line and the solid black curve, where the region of distribution independent stability lies between the solid and dashed gray lines. Substituting the approximations using moments, i.e. equations (25), into (15) we get approximations to the true boundary of stability depicted as the dotted and dash lines in Figures 4(a) and 4(b). The approximations using cumulants are obtained by substituting (27) and (28) into (15). In Figures 4(a) and 4(b), the first and second approximations using cumulants are represented by the curves depicted by crosses and circles, respectively.

We note that for the uniform distribution (Figure 3) and for the gamma distribution (Figure 4), the approximations using cumulants give better results than the approximations using moments. This is expected since for the approximations using cumulants, the truncation in the expansion occurs inside the exponential, sine and cosine functions, therefore we expect better numerical results. As a general rule, all approximations improve as more moments or cumulants are added. For all cases, the second approximation using cumulants lies very close to the true boundary of stability. The first approximation using moments enters the distribution independent region in all four cases and the second approximation using moments also enters this region in case of the uniform distribution with $\rho=1$. No approximation using cumulants enters the distribution independent region. We also notice that all approximations give good results for large negative values of $z_{k}$. 


\section{Connection Matrix with Complex Eigenvalues}

In this section we analyze the stability of the equilibrium point $\mathbf{v}^{*}$ of (6), for a general interconnection matrix $W$. In this case, the eigenvalues of $W$ can be complex, $z_{k}=a_{k}+i b_{k}$, with $a_{k}, b_{k} \in \mathbb{R}$. We will determine conditions on these eigenvalues, in terms of the model parameters $\alpha, \beta$ and $\tau$, that guarantee that the equilibrium point is locally asymptotically stable or unstable.

With definition of $z_{k}$ above, the characteristic equation (12) becomes

$$
0=\Delta(\lambda)=\prod_{k=1}^{n} \Delta_{k}(\lambda)=\prod_{k=1}^{n}\left(\lambda+\alpha \tau-\beta \tau\left(a_{k}+i b_{k}\right) \int_{0}^{\infty} \hat{g}(v) e^{-\lambda v} d v\right)
$$

where $\alpha, \beta>0$. To determine the stability region we need to determine conditions such that all roots of (34) have negative real parts.

Theorem 3. In the limit $\tau \rightarrow 0$, the equilibrium point $\mathbf{v}^{*}$ of (6) is locally asymptotically stable if $a_{k}<\alpha / \beta$ for $k=1,2, \ldots, n$.

Proof. As mentioned in Section 1, when $\tau \rightarrow$ 0, the linearized model with delay (8) approaches the non-delayed linerized model (9). Now the characteristic equation of (9) is

$$
0=\prod_{k=1}^{n}\left(\lambda+\alpha-\beta\left(a_{k}+i b_{k}\right)\right)
$$

Thus all roots of the characteristic equation have negative real parts if $a_{k}<\alpha / \beta$ for $k=1,2, \ldots, n$. The result follows.

Now consider the case $\tau>0$. We will study the roots of (34) by considering the roots of each factor, $\Delta_{k}(\lambda)$. From Section 2, we know that if $b_{k}=0$ and $\left|a_{k}\right|<\alpha / \beta$ then all roots of $\Delta_{k}(\lambda)$ have negative real parts. Further, a standard result $[25,41]$ indicates that as the coefficients of $\Delta_{k}(\lambda)$ are varied, the number of roots with positive real parts can only change by a root passing through the imaginary axis. Now $\lambda=0$ is a root of $\Delta_{k}(\lambda)$ only when $a_{k}=\alpha / \beta$ and $b_{k}=0$. Further, using the definitions of $C(\omega)$ and $S(\omega)$ in (14), $\lambda=i \omega$ is a root of $\Delta_{k}(\lambda)$ when $a_{k}, b_{k}, \alpha, \beta, \tau$ satisfy the following equation

$$
0=\Delta_{k}(i \omega)=i \omega+\alpha \tau-\beta \tau\left(a_{k}+i b_{k}\right)(C(\omega)-i S(\omega)) .
$$

Separating (35) into the real and imaginary parts we obtain

$$
\begin{gathered}
\alpha=\beta a_{k} C(\omega)+\beta b_{k} S(\omega), \\
-\omega=\beta \tau a_{k} S(\omega)-\beta \tau b_{k} C(\omega) .
\end{gathered}
$$

To analyze these equations we need the following basic properties of $C(\omega)$ and $S(\omega)$.

Lemma 1. Let $C(\omega)$ and $S(\omega)$ be defined as in (14). Then $C(0)=1, S(0)=0, C^{\prime}(0)=0, S^{\prime}(0)=1$ and $C^{2}(\omega)+S^{2}(\omega) \leq 1$ for any distribution.

Proof. The first four results follow directly from the definitions of $C(\omega)$ and $S(\omega)$ in (14). For the last result, note that

$$
\begin{aligned}
C^{2}(\omega) & =\left(\int_{0}^{\infty} \cos (\omega v) \hat{g}(v) d v\right)^{2} \\
& =\int_{0}^{\infty} \cos (\omega v) \hat{g}(v) d v \int_{0}^{\infty} \cos (\omega w) \hat{g}(w) d w \\
& =\int_{0}^{\infty} \int_{0}^{\infty} \cos (\omega v) \cos (\omega w) \hat{g}(v) \hat{g}(w) d w d v
\end{aligned}
$$


Similarly,

$$
S^{2}(\omega)=\int_{0}^{\infty} \int_{0}^{\infty} \sin (\omega v) \sin (\omega w) \hat{g}(v) \hat{g}(w) d w d v
$$

Adding the two expressions we get

$$
\begin{aligned}
C^{2}(\omega)+S^{2}(\omega) & =\int_{0}^{\infty} \int_{0}^{\infty} \cos (\omega v-\omega w) \hat{g}(v) \hat{g}(w) d w d v \\
& \leq \int_{0}^{\infty} \int_{0}^{\infty}|\cos (\omega v-\omega w)| \hat{g}(v) \hat{g}(w) d w d v \\
& \leq \int_{0}^{\infty} \hat{g}(v) d v \int_{0}^{\infty} \hat{g}(w) d w=1 .
\end{aligned}
$$

Now we can obtain extensions of result (1) of Theorem 1 and its corollary to the case when the eigenvalues of the connection matrix are complex.

Theorem 4. The equilibrium point $\mathbf{v}^{*}$ of (6) is locally asymptotically stable for any distribution, $g$, if $\left|z_{k}\right|<\alpha / \beta$, $k=1,2, \ldots, n$.

Proof. Squaring and adding equations (36) and Lemma 1 we obtain a necessary condition on the magnitude of $z_{k}$ for a pure imaginary root of $\Delta_{k}(\lambda)$ to exist:

$$
\begin{aligned}
\left|z_{k}\right| & =\frac{\sqrt{\alpha^{2} \tau^{2}+\omega^{2}}}{\beta \tau \sqrt{C^{2}(\omega)+S^{2}(\omega)}} \\
& \geq \frac{\alpha}{\beta} .
\end{aligned}
$$

Clearly this cannot be satisfied if $\left|z_{k}\right|<\alpha / \beta$, so the result follows.

Note that this region is the delay independent region of stability of the corresponding model with one fixed delay $\tau$.

Corollary 2. Let $\gamma=f^{\prime}(0)$. The equilibrium point $\mathbf{v}^{*}$ of (6) is locally asymptotically stable for any distribution, $g$, if $\left|z_{k}\right|<\alpha / \gamma, k=1,2, \ldots, n$.

These conditions are easier to check, since they only require knowledge of the neuron gain $\gamma=f^{\prime}(0)$.

To get more precise conditions for stability, we solve for $a_{k}$ and $b_{k}$ from (36),

$$
\begin{aligned}
& a_{k}=\frac{\tau \alpha C(\omega)-\omega S(\omega)}{\beta \tau\left(C^{2}(\omega)+S^{2}(\omega)\right)} \stackrel{\text { def }}{=} R(\omega), \\
& b_{k}=\frac{\tau \alpha S(\omega)+\omega C(\omega)}{\beta \tau\left(C^{2}(\omega)+S^{2}(\omega)\right)} \stackrel{\text { def }}{=} I(\omega) .
\end{aligned}
$$

For fixed $\alpha, \beta$ and $\tau$, equations (37) and (38) represent a curve in the complex plane parameterized by $\omega$. It is easy to check that this curve has the following properties

- It is symmetric about the $b_{k}=0$.

- When $\omega=0$, it passes through the point $(\alpha / \beta, 0)$ with infinite slope.

- It lies outside the circle $a_{k}^{2}+b_{k}^{2}=\alpha^{2} / \beta^{2}$.

Let $\bar{\omega}$ be the smallest positive value such that $I(\bar{\omega})=0$. If $I(\omega) \neq 0$ for $\omega \neq 0$, then let $\bar{\omega}=\infty$. Then we have the following distribution dependent result. 
Theorem 5. Let $\alpha, \beta$ and $\tau$ be fixed. The equilibrium point $\mathbf{v}^{*}$ of (6) is locally asymptotically stable if for each $k=1,2, \ldots, n$ the point $\left(a_{k}, b_{k}\right)$ lies inside the curve $(R(\omega), I(\omega)), \omega \in[-\bar{\omega}, \bar{\omega}]$ where $R(\omega)$ and $I(\omega)$ are defined by (37)-(38).

Proof. From Theorem 4, all roots of $\Delta_{k}(\lambda)$ have negative real parts if $\left|z_{k}\right|<\alpha / \beta$. For fixed $\alpha, \beta$ and $\tau$, this corresponds to the point $\left(a_{k}, b_{k}\right)$ lying inside the circle $a_{k}^{2}+b_{k}^{2}=\alpha^{2} / \beta^{2}$. As discussed above, if $a_{k}$ and $b_{k}$ are moved outside this circle the number of roots of $\Delta_{k}(\lambda)$ with positive real parts can only change if there is root with zero real part for some values of $a_{k}$ and $b_{k}$. From the properties of the curve given above, this will occur when $a_{k}=R(\omega), b_{k}=I(\omega)$ for some $\omega \in[-\bar{\omega}, \bar{\omega}]$. The result follows.

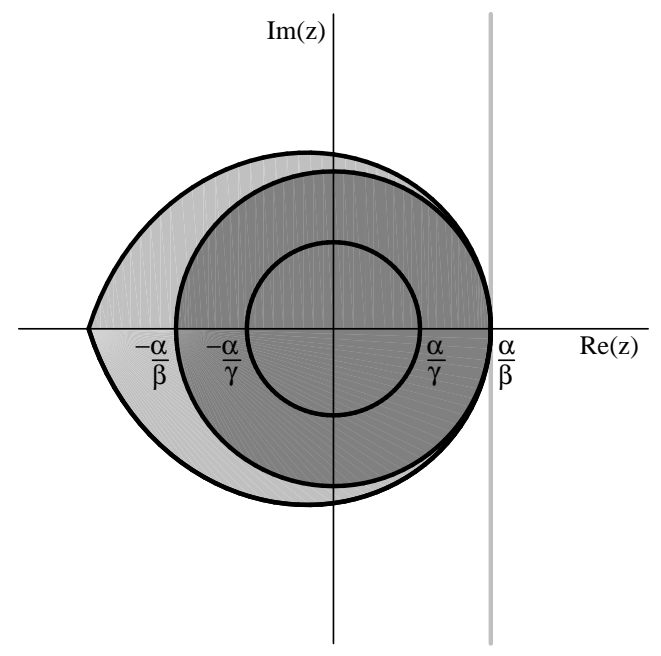

Figure 5: Illustration of the stability region described by Theorems $3-5$. The region to the left of the grey line is the stability region for $\tau \rightarrow 0$. If all eigenvalues of the connection matrix lie inside the dark gray disk with boundary $\left|z_{k}\right|=\alpha / \beta$, then the equilibrium point $\mathbf{v}^{*}$ of (10) is stable for any mean delay or any distribution. The actual stability region (the light gray tear drop region) will depend on the particular distribution and the value of the mean delay $\tau$. The region inside the smaller circle of radius $\alpha / \gamma$ also guarantees stability for any mean delay and any distribution; it is more conservative, but it is easier to obtain, since it only requires knowledge of the neuron gain $\gamma=f^{\prime}(0)$.

The results of Theorems 4 and 5 are depicted in Figure 5. As in [32], we represent the condition given by each theorem by a region in the complex plane such that if all the $z_{k}$ lie inside this region then the condition is satisfied. We will refer this region as the stability region of the equilibrium point $\mathbf{v}^{*}$ of (6). The region to the left of the grey line is the stability region for $\tau \rightarrow 0$ given by Theorem 3. The distribution independent region of stability (for $\tau>0$ ) is shown in dark gray. The larger circle shows the result of Theorem 4 and the smaller one that of Corollary 2 . The actual stability region (light grey) described by Theorem 5 encompasses this conservative region. Its shape depends on the particular distribution and the value of the mean delay.

A commonly held belief is that a system with a distribution of delays is more stable than the same system with a fixed delay. The following shows this is true for our system, for large enough mean delay.

Theorem 6. In the limit $\tau \rightarrow \infty$, the stability region of the equilibrium $\mathbf{v}^{*}$ of (6) with the Dirac distribution (i.e. a fixed delay) lies inside or is the same as the stability region of the equilibrium $\mathbf{v}^{*}$ of (6) with any other distribution.

Proof. From Theorem 5 the stability region of the equilibrium point for any distribution is the region in the complex plane enclosed by the curve $(R(\omega), I(\omega)), \omega \in[-\bar{\omega}, \bar{\omega}]$ where $R(\omega)$ and $I(\omega)$ are defined by (37)-(38). In the limit at $\tau \rightarrow \infty$ this curve is given by $\left(R_{\infty}(\omega), I_{\infty}(\omega)\right), \omega \in[-\bar{\omega}, \bar{\omega}]$ where $R_{\infty}(\omega)$ and $I_{\infty}(\omega)$ are defined by

$$
\begin{aligned}
R_{\infty}(\omega) & =\lim _{\tau \rightarrow \infty} R(\omega)=\frac{\alpha C(\omega)}{\beta\left(C^{2}(\omega)+S^{2}(\omega)\right)}, \\
I_{\infty}(\omega) & =\lim _{\tau \rightarrow \infty} I(\omega)=\frac{\alpha S(\omega)}{\beta\left(C^{2}(\omega)+S^{2}(\omega)\right)} .
\end{aligned}
$$




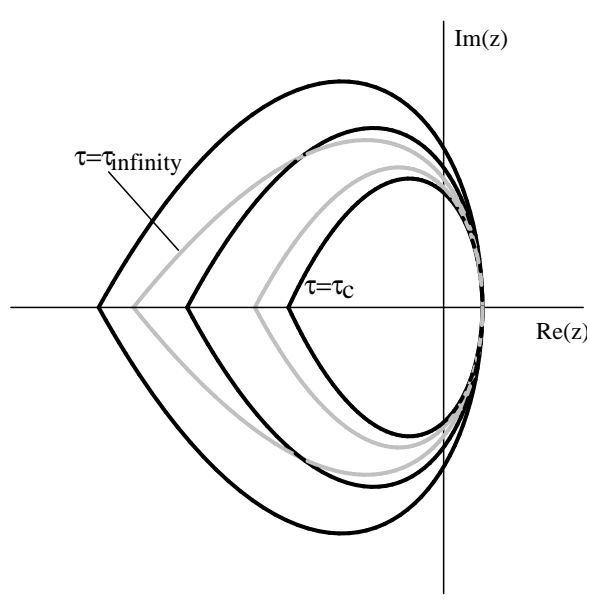

(a) Real $z_{k}$ 's

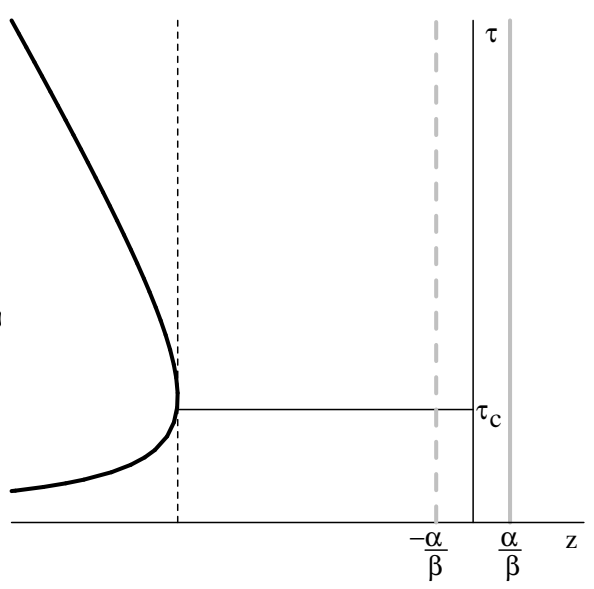

(b) Complex $z_{k}$ 's

Figure 6: (a) As $\tau$ increases, the region of stability decreases till it reaches a minimum stability region at $\tau=\tau_{c}$, represented by the most inner black curve. For $\tau>\tau_{c}$, the region of stability increases till it reaches a maximum stability region as $\tau \rightarrow \infty$, depicted by the outer gray curve. Thus if all $z_{k}, k=1, \ldots, n$, are inside the boundary corresponding to $\tau=\tau_{c}$, the equilibrium point is stable for any value of the mean delay. (b) For real eigenvalues of the connection matrix, the value of $\tau_{c}$ corresponds to the maximum on the boundary of stability (i.e. the curve has a vertical slope in the $z \tau$-plane). Thus in the region between the dash black and solid gray curve, the equilibrium is stable for any value of the mean delay.

For the case of a delta distribution, i.e. a fixed delay, the stability region is as defined above, with $C(\omega)=\cos (\omega)$ and $S(\omega)=\sin (\omega)$. As $\tau \rightarrow \infty$ this region becomes the circle $|z|=\alpha / \beta$. The result then follows from the fact that

$$
R_{\infty}(\omega)^{2}+I_{\infty}(\omega)^{2}=\frac{\alpha^{2}}{\beta^{2}\left(C^{2}(\omega)+S^{2}(\omega)\right)} \geq \frac{\alpha^{2}}{\beta^{2}}
$$

We note that the behaviour of the stability region as the mean delay $\tau$ varies can be quite different for different distributions. As presented in [32], the size of the region of stability of the model with fixed delay decreases monotonically as $\tau$ increases. As $\tau \rightarrow \infty$, the stability region approaches the delay independent region of stability, $\left|z_{k}\right|<\alpha / \beta$. For the model with other distributions, we do not necessarily have this uniform convergence as the mean delay becomes larger and larger. For example, for the gamma distribution with $p=2$, there exists a particular value of the mean delay, $\tau=\tau_{c}$, such that if all eigenvalues of the connection matrix are inside the tear drop region given by

$$
\left|z_{k}\right|=\frac{\sqrt{\alpha^{2} \tau_{c}^{2}+\omega^{2}}}{\beta \tau_{c} \sqrt{C^{2}(\omega)+S^{2}(\omega)}},
$$

then the equilibrium point is locally asymptotically stable for any value of the mean delay. But unlike the model with fixed delay, the value of $\tau_{c}$ is not infinity. This is depicted in Figure 6(a). As $\tau$ increases the region of stability decreases until it reaches a minimum stability region at $\tau=\tau_{c}$, represented by the inner most black curve. For $\tau>\tau_{c}$, the region of stability increases till it reaches a maximum stability region as $\tau \rightarrow \infty$, depicted by the outer gray curve. Hence stability can be regained as we increase the mean delay, which cannot be achieved for the model with fixed delay. For real eigenvalues of the connection matrix, the value of $\tau_{c}$ corresponds to the maximum of the boundary of stability. As seen in Figure 6(b), at this maximum, the boundary of stability has a vertical slope (depicted as the dash black line) and if all $z_{k}, k=1, \ldots, n$, are to the right of this line and less than $\alpha / \beta$, then the equilibrium point is stable for any value of the mean delay. If any of the $z_{k}$ 's are located to the left of the vertical line, the stability can be regained for sufficiently large $\tau$.

We illustrate our results with an example, where we analyze a three dimensional network with a particular connection matrix. We investigate the stability region of the equilibrium point of the nonlinear model in (6) with $n=3$ and 
of its corresponding model with a fixed delay as we vary $\beta$.

Example. Consider the connection matrix

$$
W=\left(\begin{array}{ccc}
0 & 1 & 0 \\
-65 / 64 & 0 & 1 / 16 \\
65 / 64 & 1 & 0
\end{array}\right)
$$

with eigenvalues $z_{1}=1 / 4, z_{2,3}=-1 / 8 \pm i$. In the following simulations, we let $\alpha=1$ and $F_{k}=0$ for $k=1,2,3$, and $\tau=3$ (this value of $\tau$ is in fact the critical value of the mean delay, i.e. if the three eigenvalues of $W$ are inside the region given by (39) with $\tau_{c}=3$, then the equilibrium point is stable for any value of the mean delay). We compare the stability region of the equilibrium point of the three dimensional nonlinear model in (6) to the stability region of the equilibrium point of the corresponding model with a fixed delay $\tau=3$. We let $g(u)$ to represent a gamma distribution with $p=3$ and the signal function to be $f(v)=\tanh (\beta v)$.

For $\beta=0.5$ the three eigenvalues of the connection matrix lie within the boundary of stability for the distributed delay model (the solid black curve) and also within the boundary of stability for the model with one fixed delay (the curve depicted by crosses), as seen in Figure 7(a). In this case, the three eigenvalues of $W$ also lie inside the delay and distribution independent region of stability (the gray circle), hence Theorem 4 or Theorem 5 predicts that the equilibria of the model with both distributed and fixed delay are stable. This is seen in Figures 7(b) and (c), where all neurons converge to the steady state solution.

For $\beta=1.2$ the three eigenvalues of the connection matrix lie within the boundary of stability for the distributed delay model (the solid black curve), but two of them lie outside the boundary of stability for the fixed delay model (the curve depicted by crosses), as seen in Figure 8(a). Thus Theorem 5 predicts that the equilibrium of the distributed delay model is stable, but cannot predict anything about the stability of the equilibrium of the fixed delay model. Figure 8(b) shows the neurons in the distributed delay model converging to the steady state solution, whereas the neurons in the fixed delay model oscillate, as seen in Figure 8(c).

For $\beta=1.5$ the three eigenvalues of the connection matrix lie outside both the boundary of stability for the distributed and fixed delay models, as seen in Figure 9(a). Thus Theorem 5 cannot be applied to predict anything about the stability of the equilibria of the two models. Figures 9(b) and (c) show the three neurons oscillating for both the distributed and fixed delay models.

We note that for $\beta=1.5$, the stability of the equilibrium point of the distributed delay model can be recovered by increasing the mean delay. As $\tau$ is increased beyond the critical value $\tau_{c}=3$, the boundary of the stability region becomes larger and eventually encompasses the three eigenvalues, as seen in Figure 10(a). When $\tau=20$, Theorem 5 predicts that the equilibrium of the distributed delay model is stable. On the other hand, for the fixed delay model, as $\tau$ becomes larger, the boundary of the stability region becomes becomes smaller and thus the stability of the equilibrium point can never be recovered. Figure 10(b) shows the neurons in the distributed delay model converging to the steady state solution, whereas the neurons in the fixed delay model oscillate, as seen in Figure 10(c).

We can obtain approximations for the boundary of the stability region, $(R(\omega), I(\omega)) \omega \in[-\bar{\omega}, \bar{\omega}]$, by using the approximations for $C(\omega)$ and $S(\omega)$ derived in subsection 2.2. In the following subsections we compare these approximations with the exact boundary for the uniform and gamma distributions.

\subsection{Verifying the approximations for the uniform distribution}

In this section we plot the true region of stability for the uniform distribution in order to compare it to the approximations using the moments and the cumulants. From (31) we obtain

$$
C^{2}(\omega)+S^{2}(\omega)=\frac{4}{\rho^{2} \omega^{2}} \sin ^{2}\left(\frac{\rho \omega}{2}\right) .
$$

Substituting this and the exact expression for $C(\omega)$ and $S(\omega)$ from (31) into (37) and (38) we obtain

$$
\begin{aligned}
a_{k} & =\frac{\rho \omega(\tau \alpha \cos (\omega)-\omega \sin (\omega))}{2 \beta \tau \sin \left(\frac{\omega \rho}{2}\right)}, \\
b_{k} & =\frac{\rho \omega(\tau \alpha \sin (\omega)+\omega \cos (\omega))}{2 \beta \tau \sin \left(\frac{\omega \rho}{2}\right)} .
\end{aligned}
$$




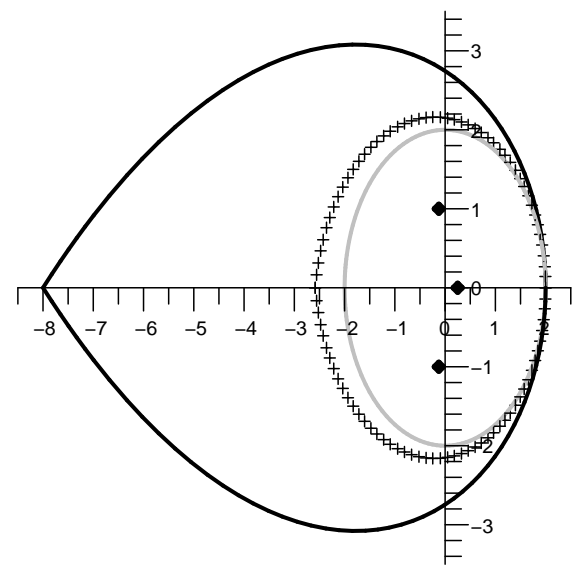

(a) $\beta=0.5(\tau=3, \alpha=1)$

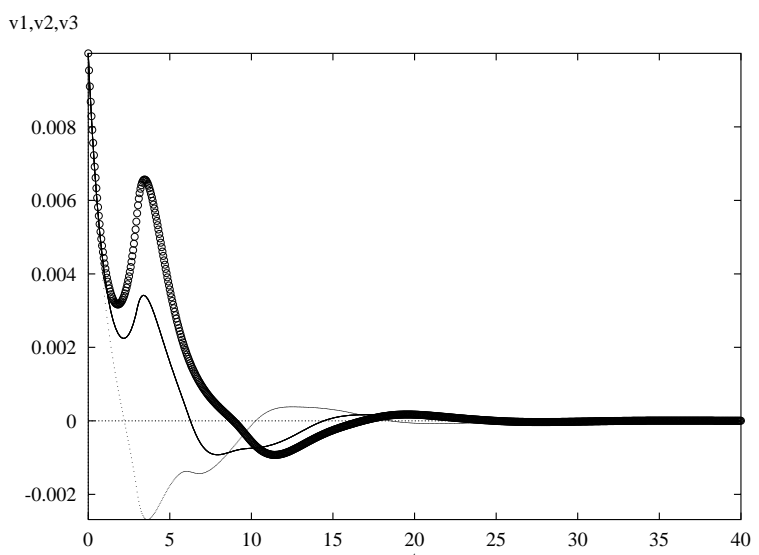

(b) Distributed delay (gamma, $p=3$ )

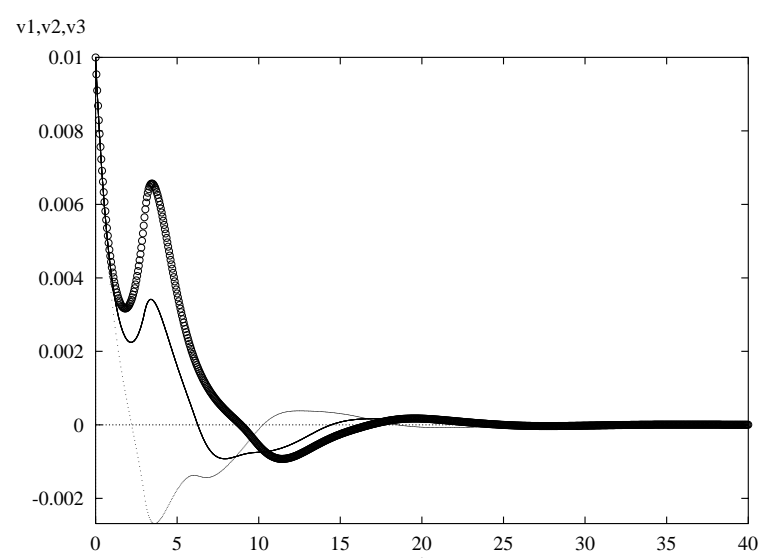

(c) Fixed delay

Figure 7: (a) The stability boundary of the distributed delay model is given by the black curve and of the model with fixed delay by the curve depicted by crosses. The gray circle represents the delay and distribution independent region of stability given by Theorem 4 . The eigenvalues of the connection matrix are plotted as dots. Theorem 4 or Theorem 5 predicts that, for $\beta=0.5$, the equilibrium of the model is stable for both distributed and fixed delays. This is confirmed by numerical simulations: all three neurons converge to the steady state solution in the model with (b) distributed and (c) fixed delay . 


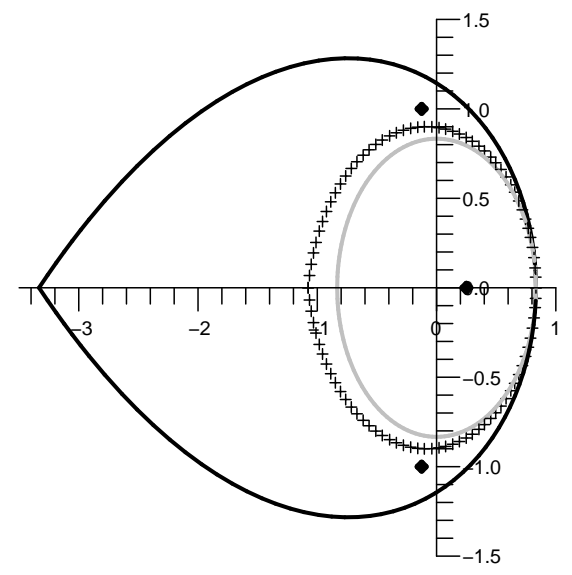

(a) $\beta=1.2(\tau=3, \alpha=1)$

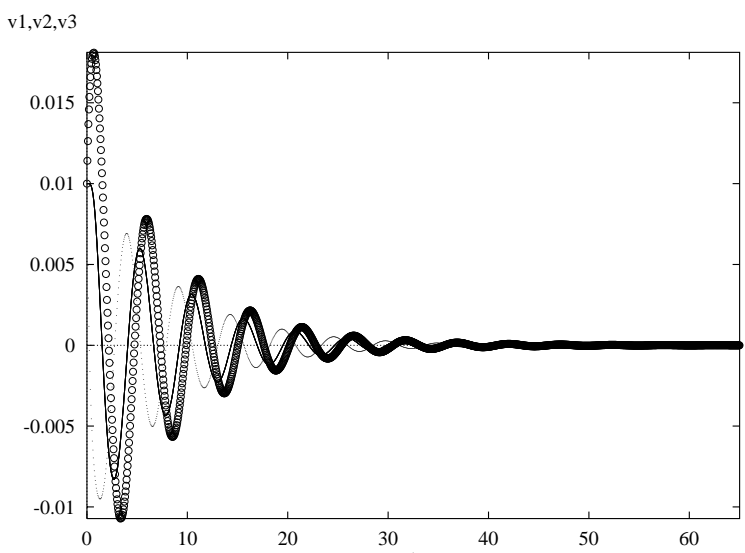

(b) Distributed delay (gamma, $p=3$ )

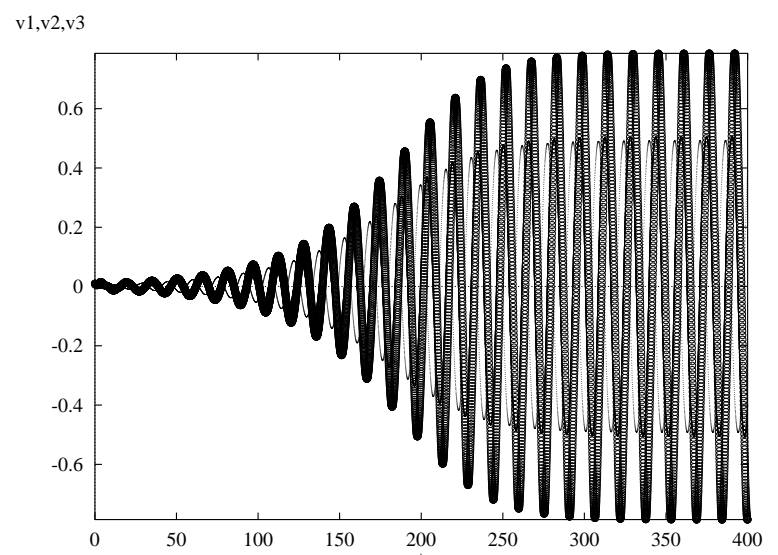

(c) Fixed delay

Figure 8: (a) The stability boundary of the distributed delay model is given by the black curve and of the fixed delay model by the curve depicted by crosses. The gray circle represents the delay and distribution independent region of stability given by Theorem 4 . The eigenvalues of the connection matrix are plotted as dots. Theorem 5 predicts that, for $\beta=1.2$, the equilibrium of the distributed delay model is stable, but cannot predict anything about the stability of the equilibrium point of the fixed delay model. This is confirmed by numerical simulations: (b) all three neurons in the distributed delay model converge to the steady state solution; (c) all three neurons in the fixed delay model oscillate. 


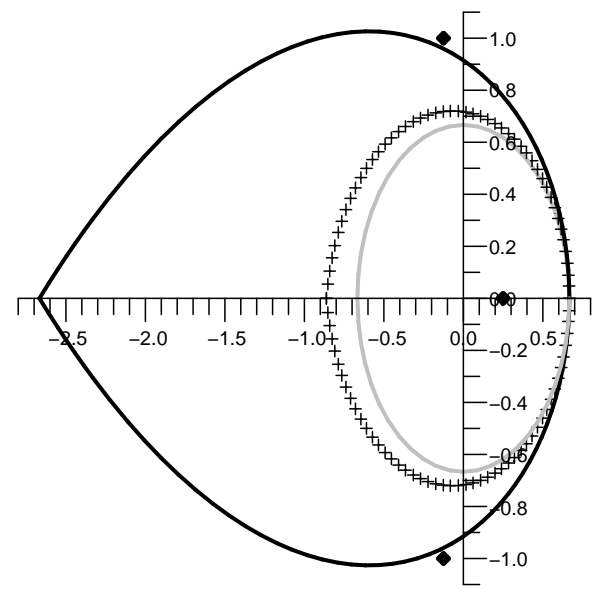

(a) $\beta=1.5(\tau=3, \alpha=1)$

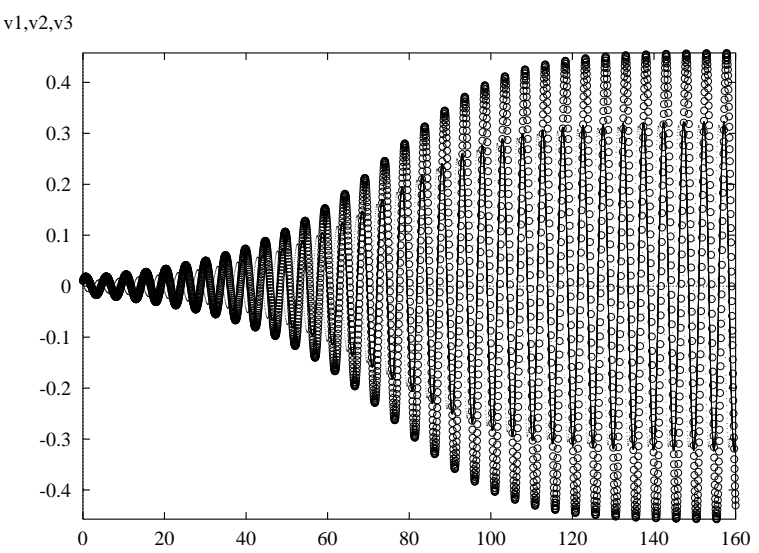

(b) Distributed delay (gamma, $p=3$ )

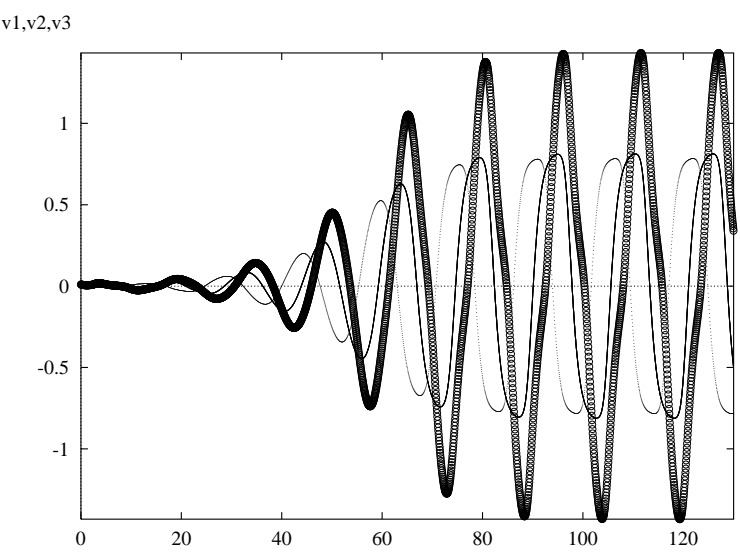

(c) Fixed delay

Figure 9: (a) The stability boundary of the distributed delay model is given by the black curve and of the fixed delay model by the gray curve. The eigenvalues of the connection matrix are plotted as dots. For $\beta=1.8$, Theorem 4 cannot be applied to predict the stability of the equilibria of the distributed and fixed delay models. This is confirmed by numerical simulations: all three neurons in the (b) distributed and (c) fixed delay models oscillate. 


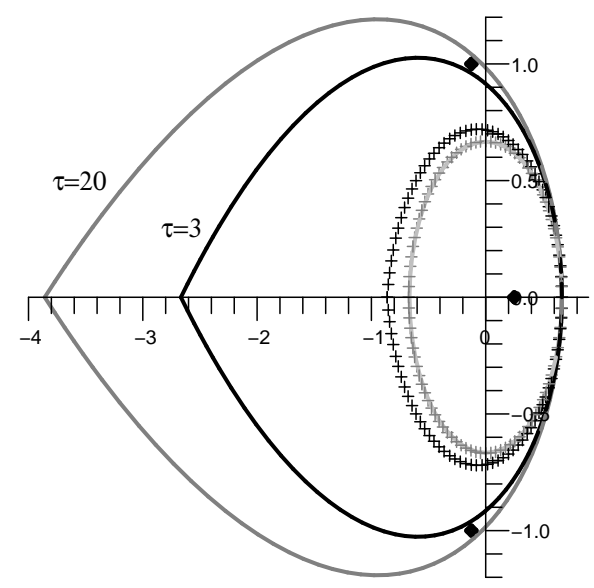

(a) $\beta=1.5(\tau=20, \alpha=1)$

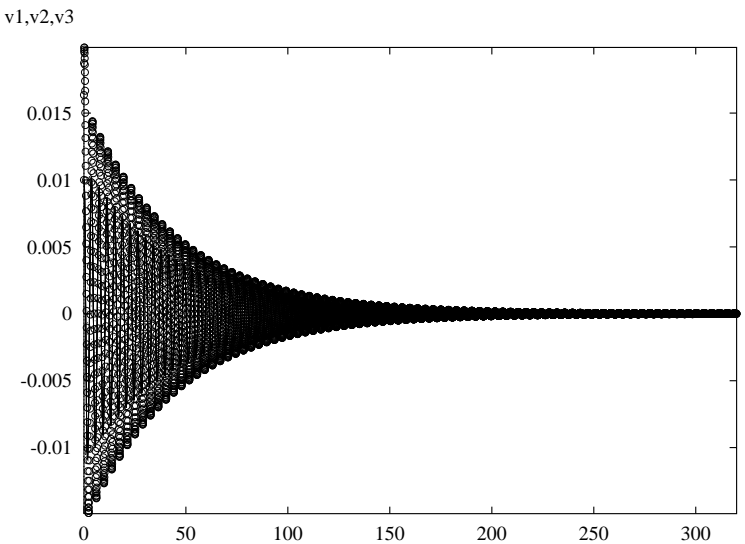

(b) Distributed delay ('gamma, $p=3$ )

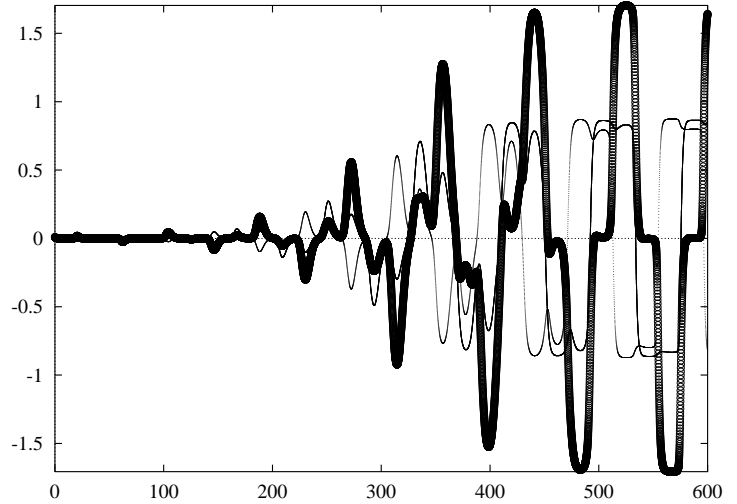

(c) Fixed delay

Figure 10: (a) For the distributed delay model, the boundary of the stability when $\tau=20$ is represented by the dark gray curve, and for $\tau=3$ by the black curve. When $\tau=20$, the boundary of stability is larger than the one when $\tau=3$, encompassing the three eigenvalues of $W$, and hence the stability of the equilibrium point of the distributed delay model is recovered by increasing the delay. For the fixed delay model, the boundary of the stability when $\tau=20$ is represented by the gray crosses, and for $\tau=3$ by the black crosses. When $\tau=20$, the boundary of stability is smaller than the one when $\tau=3$, thus the stability of the equilibrium point of the fixed delay model is never recovered by increasing the delay. This is confirmed by numerical simulations: when $\tau=20$, (b) all three neurons in the distributed delay model converge to the steady state solution; (c) all three neurons in the fixed delay model oscillate. 


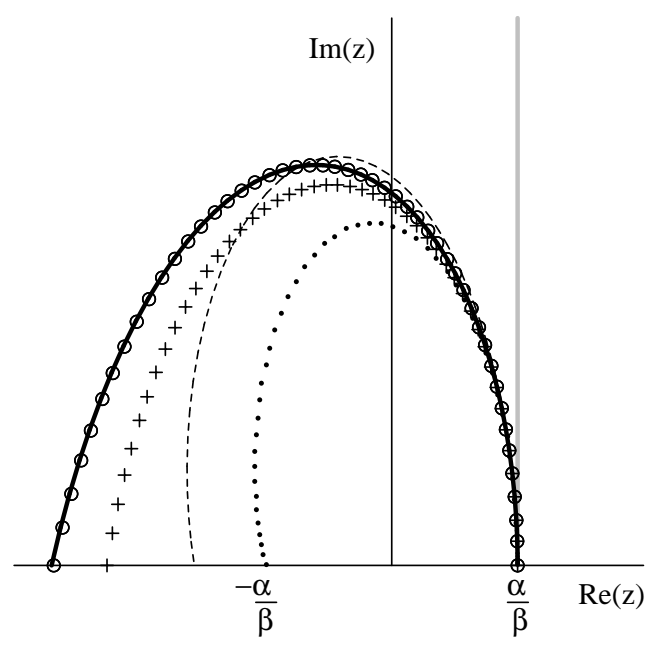

(a) $\rho=1$

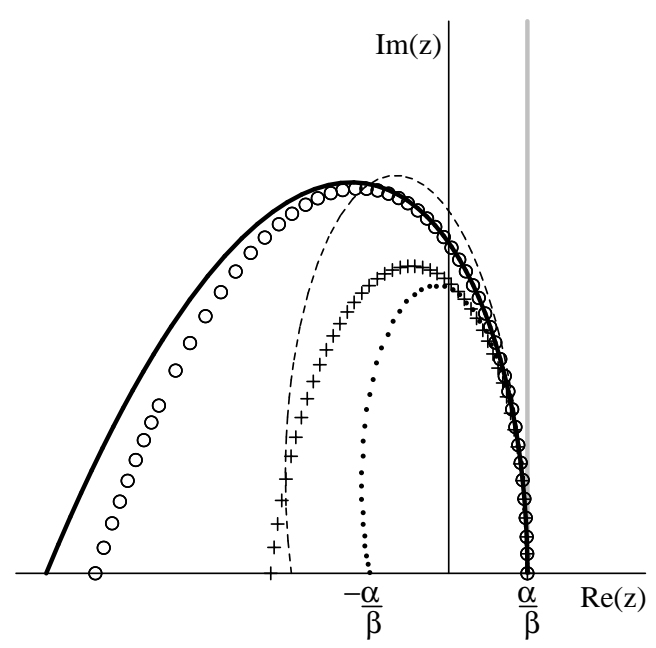

(b) $\rho=2$

Figure 11: Stability region for the uniform distribution with $\rho=1$ and $\rho=2$ when $\tau=1 / 2$. The true region of stability lies between the solid black black and the real-axis. The dotted and dash curves show the first and second approximations using the moments, respectively. The first and second approximations using cumulants are given by the curves depicted as crosses and circles, respectively. Without delay, the stability region lies to the left of the solid gray line.

The above two equations represents the true boundary of stability in the case of the uniform distribution, and it is represented by the solid black line in Figure 11(a) for $\rho=1$, and in Figure 11(b) for $\rho=2$.

Substituting (25) into (37) and (38), we obtain the approximate stability boundary using the moments of the uniform distribution. In Figure 11, the first and second approximations using the moments are represented by the dotted and dash curves, respectively. Substituting (27) and (28) into (37) and (38), we obtain the approximate boundaries of stability using cumulants. The first and second approximations using cumulants are seen in Figure 11 as the curves depicted by crosses and circles, respectively.

\subsection{Verifying the approximations for the gamma distribution}

In this section we plot the true region of stability for the gamma distribution in order to compare it to the approximations using the moments and the cumulants. First, we notice that from (33) we have

$$
C(\omega)^{2}+S^{2}(\omega)=\left(1+\frac{\omega^{2}}{p^{2}}\right)^{-2 p}\left[\operatorname{Re}^{2}\left(1-\frac{i \omega}{p}\right)^{p}+\operatorname{Im}^{2}\left(1-\frac{i \omega}{p}\right)^{p}\right]=\left(1+\frac{\omega^{2}}{p^{2}}\right)^{-p}
$$

Substituting this and (33) into (37) and (38) we obtain

$$
\begin{aligned}
& a_{k}=\frac{\alpha}{\beta} \operatorname{Re}\left(1-\frac{i \omega}{p}\right)^{p}-\frac{\omega}{\beta \tau} \operatorname{Im}\left(1-\frac{i \omega}{p}\right)^{p}, \\
& b_{k}=\frac{\alpha}{\beta} \operatorname{Im}\left(1-\frac{i \omega}{p}\right)^{p}+\frac{\omega}{\beta \tau} \operatorname{Re}\left(1-\frac{i \omega}{p}\right)^{p} .
\end{aligned}
$$

The above two equations represents the true boundary of stability in the case of the gamma distribution, and it is represented by the solid black line in Figure 12(a) for $p=2$, and in Figure 12(b) for $p=3$. Substituting (25) into (37) and (38), we obtain the approximate stability boundary using moments. The approximations to the boundary of stability using cumulants are obtained by substituting (27) and (28) into (37) and (38). In Figure 12, the first and second approximations using the moments are represented by the dotted and dash curves, respectively, and the first and second approximations using cumulants are given by the curves depicted as crosses and circles, respectively. 


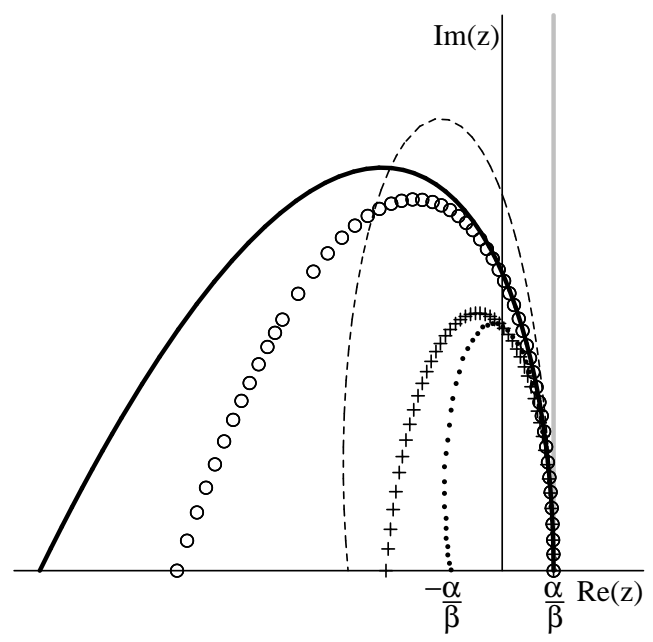

(a) $p=2$

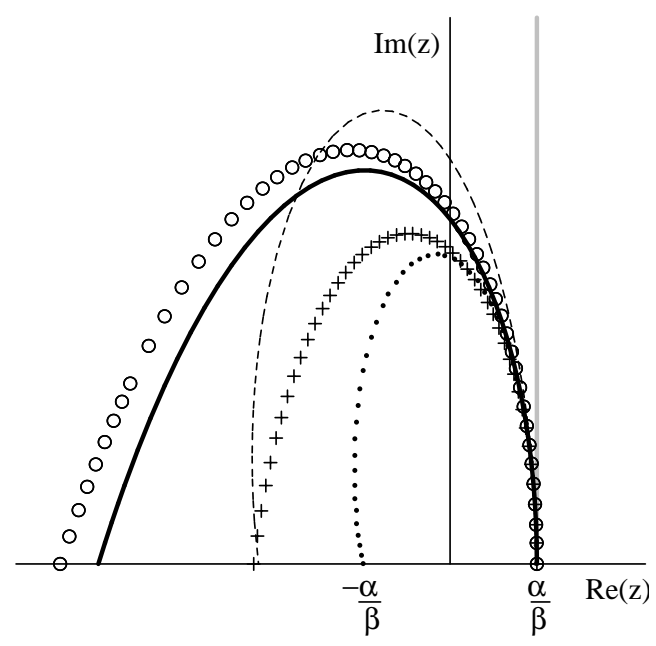

(b) $p=3$

Figure 12: Stability region for the gamma distribution with $p=2$ and $p=3$ when $\tau=1 / 2$. The true region of stability lies between the solid black black and the real-axis. The dotted and dash curves show the first and second approximations using the moments, respectively. The first and second approximations using cumulants are given by the curves depicted as crosses and circles, respectively. Without delay, the stability region lies to the left of the solid gray line.

We note that for both the uniform and gamma cases, the approximations improve as more moments or cumulants are added, and that the second approximation using cumulants lies very close to the true boundary of stability. We again notice that the approximations using cumulants give better results than the ones using moments. In Figure 13 we plot the true boundary of stability (for the uniform distribution with $\rho=1$ ) for different values of $\tau$. In contrast with Figure 6 which plots the stability region for the gamma distribution for different values of $\tau$, we can see that for the uniform distribution, the true stability region and the approximations of the stability region decrease as the mean delay $\tau$ increases. In Figure 13(a) we show a comparison between the true region of stability and the first approximations using moments and how they behave as $\tau$ increases. In Figure 13(b) we compare the true region of stability and the first approximations using cumulants for different values of $\tau$. It is again confirmed that the approximations using cumulants give better results. We note again that the first approximation using cumulants recovers the stability results of the corresponding model with fixed delay $\tau$. Hence in Figure 13(b) also shows a comparison between the stability regions of the distributed and fixed delay models for different values of $\tau$.

\section{Conclusions}

In this paper, we investigated the linear stability of the $n$ dimensional neural network with identical neurons via the analysis of the characteristic equation. When the connection matrix is symmetric, we showed that the conditions for stability are just the generalization to $n$ dimensions of the results in [6]. When the connection matrix is not necessarily symmetric, its eigenvalues $z_{k}$ may be complex and we formulated conditions on $z_{k}$ for the linear stability of the equilibrium point of (10). Our main results are outlined below.

We obtained several distribution independent results. For the case of a symmetric connection matrix, we obtained a conservative region of stability which is independent of the properties of the distribution save the mean delay, and guarantees the linear stability of the equilibrium point of (10). For the case of a general connection matrix, we determined the region of stability as $\tau \rightarrow 0$ for any distribution, and showed that in the limit $\tau \rightarrow \infty$, the region of stability of the distributed delay model is always greater or equal to the region of stability of the fixed delay model. We also obtained a conservative region of stability for any value of the mean delay and for any distribution, which coincides with the delay independent region of stability for the fixed delay model. 


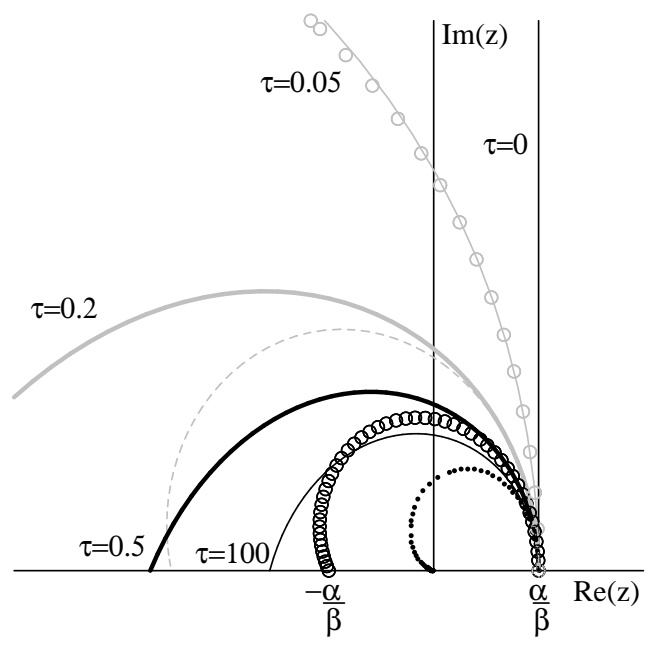

(a) Moments

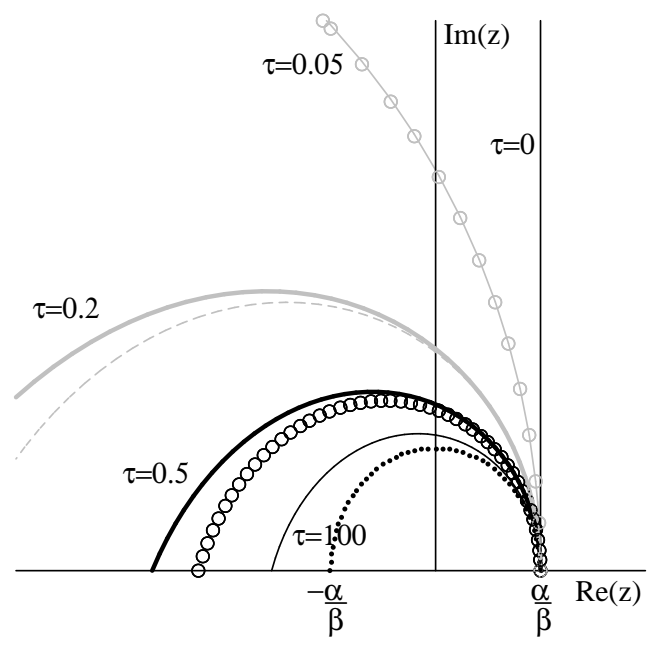

(b) Cumulants

Figure 13: The stability region for the uniform distribution with $\rho=1$ for different values of $\tau$ is compared to the first approximation using moments in (a) and to the first approximation using cumulants in (b). Without delay, the stability region lies to the left of the black line $z_{k}=\alpha / \beta$. The true boundary of stability is depicted as the thin gray line, the thick gray line, the thick black line, and the thin black line for $\tau=0.05, \tau=0.2, \tau=0.5$, and $\tau=100$, respectively. The first approximations using moments or cumulants are shown as gray circles, gray dash line, black circles, and black dotted line for $\tau=0.05, \tau=0.2, \tau=0.5$, and $\tau=100$, respectively.

Our distribution independent results compare favorably with others found in the literature. To see this, we compare our mean independent stability result described by Theorem 4 with an equivalent result obtained in [13] using Liapunov functionals. In their paper, without assuming that the connection matrix is symmetric or that neurons are identical, Gopalsamy and He find sufficient conditions that guarantee the existence and stability of a global attractor for systems of the form (3) and (5) with a gamma distributed delay. For our model, their condition translates into

$$
\|W\|_{\infty}=\max _{1 \leq k \leq n} \sum_{j=1}^{n}\left|w_{k j}\right|<\frac{\alpha}{\beta},
$$

where $\|W\|_{\infty}$ represents the maximum row sum matrix norm of $W$. Whereas, our conservative mean delay and distribution independent stability region given by Theorem 4 is

$$
\rho(W)=\max _{1 \leq k \leq n}\left|z_{k}\right|<\frac{\alpha}{\beta}
$$

where $\rho(W)$ is called the spectral radius of $W$. But by Theorem 5.6 .9 from [23] we have that $\|W\|_{\infty} \leq \rho(W)$ for any matrix $W$. Therefore the stability result using Liapunov functionals from [13] always gives a stronger, but more conservative result than our result in Theorem 4. We illustrate this through our example presented in Section 3 . In this case, $\|W\|_{\infty} \approx 2$ and $\rho(W) \approx 1$. Using (40) we cannot conclude anything about the stability of the equilibrium point for values of $\beta$ greater than 0.5 . Whereas our most conservative result guarantees stability for $\beta<1$.

Our distribution independent results are all conservative estimates of the true stability region, thus we gave a general formulation for this boundary region. See equation (15) for the symmetric connection matrix case and Theorem 5 for the general connection matrix case. Using examples, we showed that the variation of boundary of the stability region as the mean delay varies can be quite different for different distributions. There exists a particular value of the mean delay $\tau=\tau_{c}$, such that if all eigenvalues of the connection matrix are inside the boundary of stability with $\tau=\tau_{c}$, then the equilibrium point is stable, but unlike the fixed delay model, the value of $\tau_{c}$ is not necessarily infinity for other distributions.

Finally, for both symmetric and general connection matrix, we showed how the boundary of the region of stability can be approximated using the first few moments or cumulants of the distribution. The first approximation using 
cumulants always recovers the results of the corresponding model with one fixed delay. The approximations give good results when compared to the true region of stability of the equilibrium point of the uniform and gamma distributed

models. We found that the approximations using cumulants always give better results than the approximations using moments, and that the approximations improve as more cumulants or moments are added.

\section{Acknowledgements}

SAC acknowledges support of NSERC.

\section{References}

[1] M. Adimy, F. Crauste, and S. Ruan. A mathematical study of the hematopoiesis process with applications to chronic myelogenous leukemia. SIAM J. Appl. Math., 65(4):1328-1352, 2005.

[2] M. Adimy, F. Crauste, and S. Ruan. Stability and Hopf bifurcation in a mathematical model of pluripotent stem cell dynamics. Nonlin. Anal.: Real World Appl., 6:651-670, 2005.

[3] J. Arino and P. van den Driessche. Time delays in epidemic models: Modeling and numerical considerations. In Delay Diff. Eqs. and Appl., chapter 13, pages 539-558. Springer, Dordrecht, 2006.

[4] L. Berezansky and E. Braverman. Linearized oscillation thoery for a nonlinear equation with distributed delay. Mathem. and Comp. Model., 48:287-304, 2008.

[5] S. Bernard, J. Bélair, and M.C. Mackey. Sufficient conditions for stability of linear differential equations with distributed delay. DCDS, 1B:233-256, 2001.

[6] S.A. Campbell and R. Jessop. Approximating the stability region for a differential equation with a distributed delay. Math. Model. Nat. Phenom., 4(2):1-27, 2009.

[7] Y. Chen. Global stability of neural networks with distributed delays. Neur. Net., 15:867-871, 2002.

[8] M. Cohen and S. Grossberg. Absolute stability of global pattern formation and parallel memory storage by competitive neural networks. IEEE Trans. Systems Man Cybernet., 13(5):815-826, 1983.

[9] J.M. Cushing. Integrodifferential Equations and Delay Models in Population Dynamics, volume 20 of Lecture Notes in Biomathematics. Springer-Verlag, Berlin; New York, 1977.

[10] L.E. El'sgol'ts and S.B. Norkin. Introduction to the Theory and Applications of Differential Equations with Deviating Arguments, volume 105 of Mathematics in Science and Engineering Series. Academic Press, New York, 1977.

[11] T. Faria and J.J. Oliveira. Local and global stability for Lotka-Volterra systems with distributed delays and instantaneous negative feedbacks. J. Diff. Eqs., 244:1049-1079, 2008.

[12] K. Gopalsamy. Stability and oscillations in delay differential equations of population dynamics. Kluwer, Dordrecht, 1992.

[13] K. Gopalsamy and X.-Z. He. Stability in asymmetric Hopfield nets with transmission delays. Physica D, 76:344$358,1994$.

[14] F. Grégorie-Lacoste. Stabilité d'un réseau de neurones à délai distribué modélisant la mémoire spatiale. Master's Thesis, University of Montreal, 2006.

[15] S. Grossberg. Nonlinear difference-differential equations in prediction and learning theory. Proc. Nat. Acad. Sci. U.S.A., 58:1329-1334, 1967. 
[16] S. Grossberg. Global ratio limit theorems for some nonlinear functional-differential equations. I, II. Bull. Amer. Math. Soc., 74:95-99; 100-105, 1968.

[17] S. Grossberg. Some nonlinear networks capable of learning a spatial pattern of arbitrary complexity. Proc. Nat. Acad. Sci. U.S.A., 59(2):368-372, 1968.

[18] S. Grossberg. Some physiological and biochemical consequence of physiological postulates. Proc. Nat. Acad. Sci. U.S.A., 60(3):758-765, 1968.

[19] G.C. Wake H. Rasmussen and J. Donaldson. Analysis of a class of distributed delay logistic differential equations. Mathematical and Computer Modelling, 38:123-132, 2003.

[20] R. Hinch and S. Schnell. Mechanism equivalence in enzyme-substrate reactions: distributed differential delay in enzyme kinetics. Journal of Mathematical Chemistry, 35(3):253-264, 2004.

[21] J.J. Hopfield. Neurons with graded response have collective computational properties like those of two-state neurons. Proc. Natl. Acad. Sci. Biophys., 81:3088-3092, 1984.

[22] Y. Horikawa and H. Kitajimaa. Duration of transient oscillations in ring networks of unidirectionally coupled neurons. Physica D, 238(2):216-225, 2009.

[23] R.A. Horn. Matrix Analysis. Cambridge University Press, Cambridge, 1985.

[24] C. Jordan. Calculus of Finite Differences. AMS Chelsea Publishing, New Jersey, 1965.

[25] V.B. Kolmanovskii and V.R. Nosov. Stability of functional differential equations, volume 180 of Mathematics in Science and Engineering. Academic Press, London, England, 1986.

[26] Y. Kuang. Delay differential equations: with applications in population dynamics. Academic Press, Boston, 1993.

[27] B. Li, G.S.K. Wolkowicz, and Y. Kuang. Global asymptotic behaviour of a chemostat model with two perfectly complementary rersources and distributed delay. SIAM J. Appl. Math., 60:2058-2086, 2000.

[28] T. Li, Q. Luo, C. Sun, and B. Zhang. Exponential stability of recurrent neural networks with time-varying discrete and distributed delays. Nonlin. Anal.: Real World Appl., 10(4):2581 - 2589, 2009.

[29] X. Liao, K.-W. Wong, and Z. Wu. Bifurcation analysis on a two-neuron system with distributed delays. Physica D, 149:123-141, 2001 .

[30] N. MacDonald. Time lags in biological models, volume 27 of Lecture notes in biomathematics. Springer-Verlag, Berlin; New York, 1978.

[31] N. MacDonald. Biological Delay Systems: Linear Stability Theory. Cambridge University Press, Cambridge, 1989.

[32] C.M. Marcus and R.M. Westervelt. Stability of analog neural networks with delay. Phys. Rev. A, 39(1):347-359, 1989.

[33] S. Mohamad, K. Gopalsamy, and H. Akca. Exponential stability of artificial neural networks with distributed delays and large impulses. Nonlin. Anal.: Real World Appl., 9(3):872-888, 2008.

[34] C.L. Nikias and A.P. Petropulu. Higher-order Spectra Analysis. PTR Prentice Hall, New Jersey, 1993.

[35] J. Park. On global stability criterion of neural networks with continuously distributed delays. Chaos, Solitons \& Fractals, 37(2):444 - 449, 2008.

[36] S. Ruan. Delay differential equations for single species dynamics. In Delay Differential Equations and Applications, chapter 11, pages 477-515. Springer, Dordrecht, 2006. 
[37] S. Ruan and R.S. Filfil. Dynamics of a two-neuron system with discrete and distributed delays. Physica D, 191:323-342, 2004.

[38] Y. Song S. Yuan and M. Han. Direction and stability of bifurcating periodic solutions of a chemostat model with two distributed delays. Chaos, Solitons \& Fractals, 21(5):1109-1123, 2004.

[39] V. Singh. Novel global robust stability criterion for neural networks with delay. Chaos, Solitons \& Fractals, 41(1):348-353, 2009.

[40] Q. Song and Z. Wang. Neural networks with discrete and distributed time-varying delays: A general stability analysis. Chaos, Solitons \& Fractals, 37(5):1538 - 1547, 2008.

[41] G. Stépán. Retarded Dynamical Systems: Stability and Characteristic Functions, volume 210 of Pitman Research Notes in Mathematics. Longman Group, Essex, 1989.

[42] W. Su and Y. Chen. Global robust stability criteria of stochastic Cohen-Grossberg neural networks with discrete and distributed time-varying delays. Communic. in Nonlin. Sci. and Numer. Simul., 14(2):520 - 528, 2009.

[43] A. Thiel, H. Schwegler, and C.W. Eurich. Complex dynamics is abolished in delay recurrent systems with distributed feedback times. Complexity, 8(1):102-108, 2003.

[44] Z. Wang, J. Fang, and X. Liu. Global stability of stochastic high-order neural networks with discrete and distributed delays. Chaos, Solitons \& Fractals, 36(2):388 - 396, 2008.

[45] G.S.K. Wolkowicz, H. Xia, and S. Ruan. Competition in the chemostat: A distributed delay model and its global asymptotic behaviour. SIAM J. Appl. Math., 57:1281-1310, 1997.

[46] G.S.K. Wolkowicz, H. Xia, and J. Wu. Global dynamics of a chemostat competition model with distributed delay. J. Math. Biol., 38:285-316, 1999.

[47] Z. Yuan, L. Huang, D. Hu, and B. Liu. Convergence of nonautonomous Cohen-Grossberg type neural networks with variable delays. IEEE Trans. on Neur. Net., 19(1):140-147, 2008. 